\title{
The Brassica napus fatty acid exporter FAX1-1 contributes to biological yield, seed oil content, and oil quality
}

\author{
Zhongchun Xiao 1,3,4, Fang Tang 1,3, Liyuan Zhang 1,3, Shengting Li ${ }^{1,3}$, Shufeng Wang ${ }^{2}$, Qiang Huo 1,3, Bo Yang 1,3,

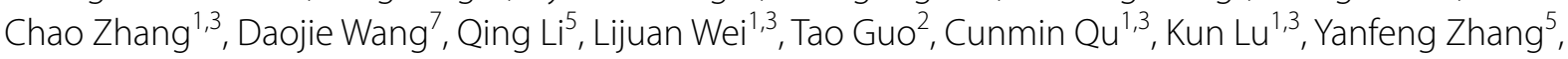 \\ Liang Guo ${ }^{6 *}$, Jiana $\mathrm{Li}^{1,3^{*}}$ and Nannan $\mathrm{Li}^{2,3^{*}}$ (D)
}

\begin{abstract}
Background: In the oilseed crop Brassica napus (rapeseed), various metabolic processes influence seed oil content, oil quality, and biological yield. However, the role of plastid membrane proteins in these traits has not been explored.

Results: Our genome-wide association study (GWAS) of 520 B. napus accessions identified the chloroplast membrane protein-localized FATTY ACID EXPORTER 1-1 (FAX1-1) as a candidate associated with biological yield. Seed transcript levels of BnaFAX1-1 were higher in a cultivar with high seed oil content relative to a low-oil cultivar. BnaFAX1-1 was localized to the plastid envelope. When expressed in Arabidopsis thaliana, BnaFAX1-1 enhanced biological yield (total plant dry matter), seed yield and seed oil content per plant. Likewise, in the field, B. napus BnaFAX1-1 overexpression lines (BnaFAX1-1-OE) displayed significantly enhanced biological yield, seed yield, and seed oil content compared with the wild type. BnaFAX1-1 overexpression also up-regulated gibberellic acid 4 (GA4) biosynthesis, which may contribute to biological yield improvement. Furthermore, oleic acid (C18:1) significantly increased in BnaFAX1-1 overexpression seeds.
\end{abstract}

Conclusion: Our results indicated that the putative fatty acid exporter BnaFAX1-1 may simultaneously improve seed oil content, oil quality and biological yield in B. napus, providing new approaches for future molecular breeding.

Keywords: Brassica napus, Fatty acid, Oil quality, Biological yield, Seed oil production

\section{Introduction}

Brassica napus (rapeseed) is an important oilseed crop for edible oils, as the oil extracted from its seeds is rich

\footnotetext{
*Correspondence: guoliang@mail.hzau.edu.cn; ljn1950@swu.edu.cn; linannan2013@swu.edu.cn

${ }^{1}$ Chongqing Engineering Research Center for Rapeseed,

College of Agronomy and Biotechnology, Southwest University,

Chongqing 400715, China

${ }^{2}$ College of Resources and Environment, and Interdisciplinary Research

Center for Agriculture Green Development in Yangtze River Basin, Southwest University, Chongqing 400715, China

${ }^{6}$ National Key Laboratory of Crop Genetic Improvement, Huazhong Agricultural University, Wuhan 430070, China

Full list of author information is available at the end of the article
}

in highly unsaturated fatty acids. In addition, rapeseed oil constitutes a reasonable substitute for diesel fuel as a renewable energy source due to their chemical similarities and high energy potential [9]. Increasing rapeseed oil production per hectare would thus increase edible oil and liquid biofuel production. Indeed, substantial work has been invested into improving $B$. napus seed yield and seed oil content. Similarly, increasing total biological yield of above-ground biomass contributes to increasing seed yield and seed oil production [30].

Previous studies revealed that rapeseed seed oil production may be enhanced by overexpressing enzymes or transcription factors involved in various metabolic 
processes. For example, the overexpression of yeast ( $\mathrm{Sac}$ charomyces cerevisiae) GLYCEROL-3-PHOSPHATE DEHYDROGENASE (GPD1) under the control of a seed-specific promoter raised seed oil content [42]. The thylakoid membrane-associated lipase, PLASTID LIPASE1 (PLIP1) was reported contributing to the export of acyl groups from plastids for seed oil biosynthesis in Arabidopsis [2, 44]. Likewise, seed oil content increased in transgenic Arabidopsis thaliana plants overexpressing B. napus WRINKLED1 (WRI1)-like [26]. The conditional expression of $B$. napus LEAFY COTYLEDON1 (BnLEC1) and LEC1-LIKE $(B n L 1 L)$ in developing seeds enhanced seed oil content by $2-20 \%$ with no detrimental effects on major agronomic traits [40]. Finally, the overexpression of B. napus GROWTH REGULATING FACTOR2 (GRF2)-like ( $B n G R F 2)$ improved seed oil production by regulating cell number and plant photosynthesis [25].

Biological yield reflects the total accumulation of photosynthetic products in plant tissues. In plants, photosynthesis and fatty acid biosynthesis take place in chloroplasts [20]. A recent study revealed that altering carbon metabolism in the chloroplasts of transgenic tobacco (Nicotiana tabacum) plants with high leaf oil levels caused biological yield and oil production to increase. We speculated that photosynthetic products transported across the chloroplast membranes may be critical for biological yield. We previously conducted a genome wide association study (GWAS) of biological yield with 520 $B$. napus accessions, and identified 88 single-nucleotide polymorphisms (SNPs) that are significantly associated with this trait [30]. Few proteins have been reported that localize to the chloroplast envelope and function as transporters regulating carbon metabolism across the chloroplast membranes. In previous work, we showed that Arabidopsis FATTY ACID EXPORTER 1 (AtFAX1) localized to the chloroplast inner envelope and mediated fatty acid export from chloroplasts, an essential step in the biosynthesis of leaf and stem lipids [21]. Subsequently, Tian et al. [41] overexpressed AtFAX1 under the control of a seed-specific promoter in Arabidopsis and demonstrated that seed-specific overexpression of AtFAX1 could increase seed size, weight, oil and protein content. Cai et al. [4] overexpressed AtFAX1 in Camelina sativa, which increased the plant biomass, seed weight, seed length, seed yield, seed oil content, and oil yield in camelina sativa. However, the function of FAX1 homologous in Brassica napus has not been reported.

To determine the contribution of chloroplast envelope proteins to biological yield, which might increase seed oil production in B. napus, we identified genes encoding proteins predicted to localize to the chloroplast envelope that mapped near significant SNPs from our previous GWAS results in B. napus. Notably, BnaFAX1-1, the $B$. napus ortholog of Arabidopsis FAX1, mapped near a significant SNP associated with biological yield. We propose that BnaFAX1-1 is a fatty acid exporter associated with biological yield in B. napus based on functional annotation analysis, subcellular localization and transcript levels across various $B$. napus cultivars. Our results revealed that BnaFAX1-1 significantly contributed to biological yield and improved seed oil production and oil quality. Furthermore, we observed that BnaFAX1-1 may modulate gibberellic acid 4 (GA4) content, and offer a potential mechanism for the increase in biological yield. The present study provides an important solution to simultaneously improve biological yield, seed oil content, seed yield and oil quality in $B$. napus by manipulating a single gene: BnaFAX1-1.

\section{Results}

Identification of chloroplast membrane-localized proteins potentially contributing to biological yield in B. napus

We previously detected SNPs that are significantly associated with biological yield during a GWAS of $520 \mathrm{~B}$. napus accessions [30]. We selected 6627 candidate genes contained within the intervals surrounding 88 significant quantitative trait loci (QTLs) associated with biological yield trait. Of those, 29 encoded proteins with potential localization to the chloroplast membrane, as determined by GO analysis (Additional file 1: Figure S1, Tables S1, S2). We further narrowed down the list of candidates to potential transporters that may be involved in the export of photosynthetic products. Notably, we identified two genes, BnaA04g02480D and BnaA07g17240D that were closely linked with the significant SNP BnA07-p12412116 [30] and encoded orthologous to the Arabidopsis membrane protein FAX1, known to mediate plastid fatty acid export.

We focused on the characterization of these two FAX1 orthologous genes. The FAX protein family consists of seven members in Arabidopsis, named AtFAX1-7 [21]. To identify potential FAX orthologues in field mustard (Brassica rapa), wild cabbage (Brassica oleracea) and $B$. napus, we performed BLAST searches, using all 7 FAX protein sequences as queries, leading to the identification of 9 putative orthologs each in B. rapa (BraFAX) and B. oleracea (BolFAX), and 21 in B. napus (BnaFAX). The physicochemical characteristics (amino acid number, theoretical isoelectric point (pI) values, relative molecular weight and number of transmembrane domains) for BnaFAX proteins are listed in Additional file 1: Table S3.

We generated an unrooted neighbor-joining phylogenetic tree based on the 46 protein sequences of FAX family members (Fig. 1A) and discovered that AtFAX1 and six putative BnaFAX1 members (BnaFAX1-1 to BnaFAX1-6) clustered into one branch. To 


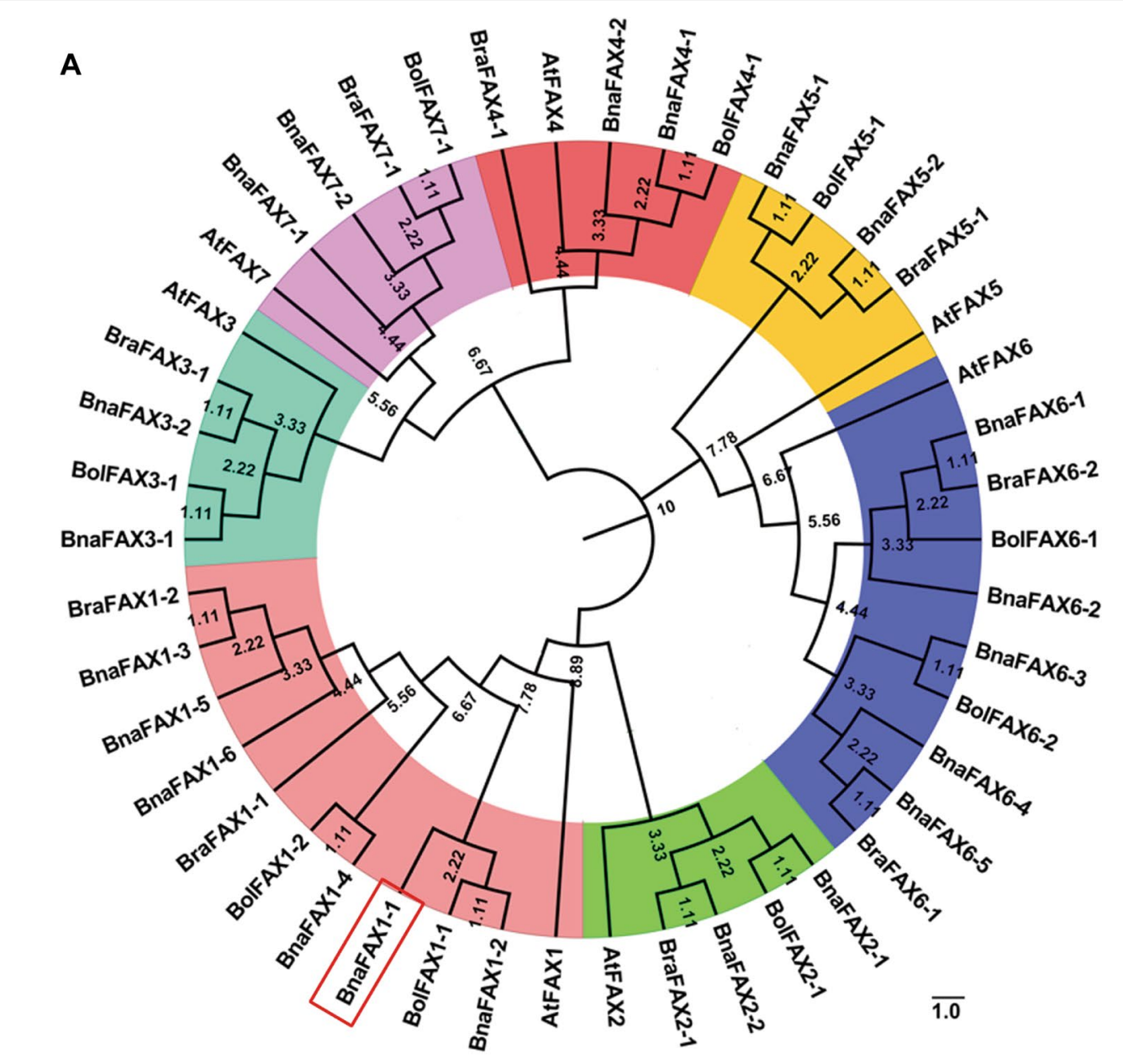

B

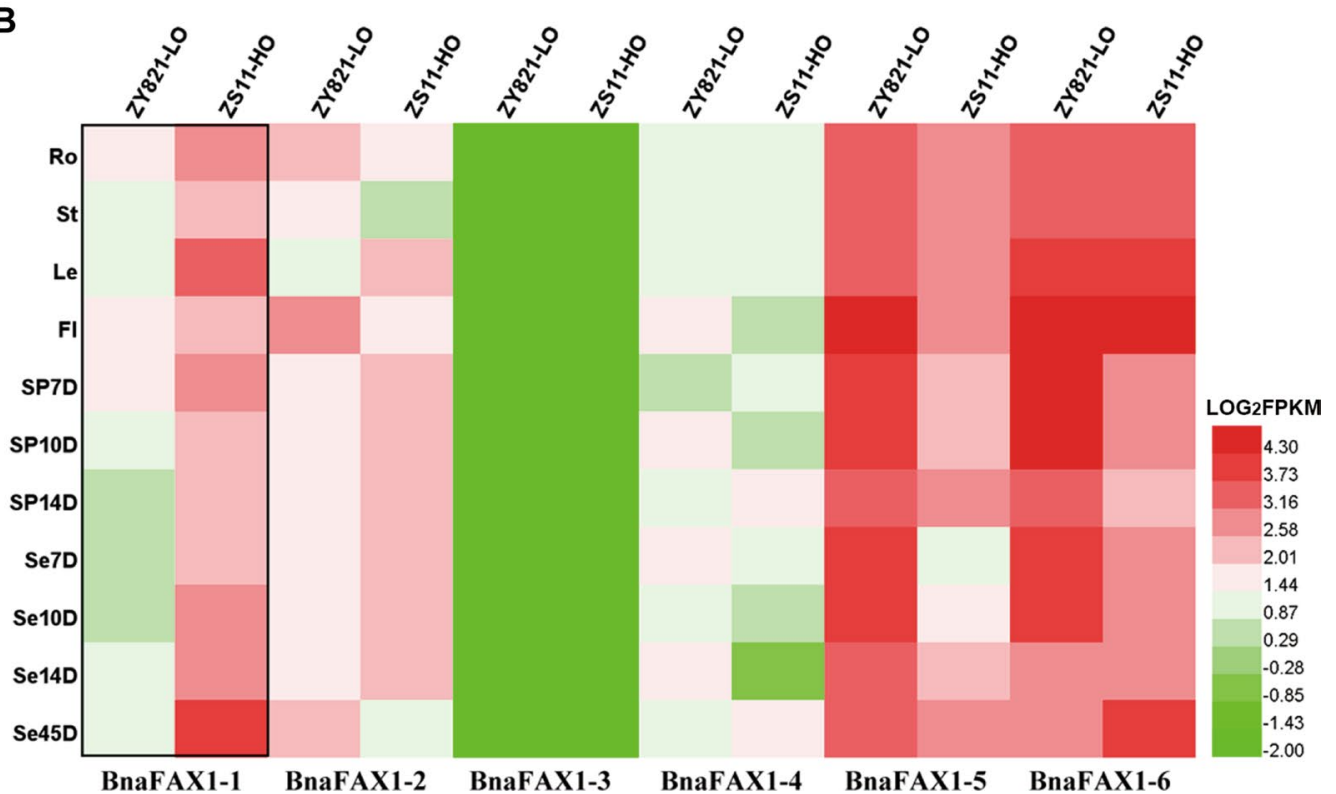

Fig. 1 Identification and analysis of new FAX genes in B. napus. A Phylogenetic analysis of FAXs in B. napus, B. rapa, B. oleracea, and A. thaliana. B Expression analysis of BnaFAX1 in different tissues collected from high-oil content (ZS11-HO) and low-oil content (ZY821-LO) B. napus genotypes. Student's $t$ test $(n=3-5 \pm S D)$. Ro: Root, St: stem, Le: leaf, FL: flower, SP: siliques pericarp, SP7D: siliques pericarp after flowering 7 days, Se: seeds, Se7D: seeds after flowering 7 days 
further characterize the B. napus FAX family, we analyzed the chromosomal locations and gene structures of the encoding genes (Additional file 1: Figure S2A, $B$ ) and predicted the conserved motifs of BnaFAX proteins using the MEME program (Additional file 1: Figure $\mathrm{S} 2 \mathrm{C}$ ). Of the six B. napus FAX1 members within the same branch as AtFAX1, BnaFAX1-1 (BnaA07g17240D) and BnaFAX1-2 (BnaCnng07490D) were the closest to AtFAX1, as evidenced by their highly similar gene structures and conserved protein motifs, suggesting that BnaFAX1-1 and BnaFAX1-2 may share the same functions as AtFAX1.

To determine potential effects of the six AtFAX1-like genes on seed oil content, we analyzed their transcript levels across various tissues in one cultivar with high seed oil contents (H, cultivar name: ZS11) and one with low seed oil content (L, cultivar name: ZY821). BnaFAX1-1 was more highly expressed in the $\mathrm{H}$ cultivar relative to the $\mathrm{L}$ cultivar in all tissues tested (Fig. 1B). By contrast, BnaFAX1-3 (BnaA04g02480D) was barely detectable in either $B$. napus cultivar. Besides, we also observed the expression levels of 6 members of BnaFAX1 in 6 tissues of a pair of high- and low-seed oil content accessions grown in Chongqing (CQ24, CQ45) and Yunnan (YN24, YN45), among which CQ24 (seed oil content about 43\%) and YN24 (seed oil content about 45\%) are high-seed oil content (H-SOC) accessions, and CQ45(seed oil content about35\%) and YN45 (seed oil content about 37\%) are low-seed oil content (L-SOC) accessions. As shown in Additional file 1: Figure S3, the expression level of BnaFAX1-1 in H-SOC accessions (CQ24, YN24) is higher than that of L-SOC accessions (CQ45, YN45) in the stem (St), leaf (Le), silique pericarps (30ZP) and seeds (30ZS) on the main inflorescence of 30 days after flowering and on the primary branch (30CP and 30CS) (Additional file 1: Figure S3A, C). This further confirms the conjecture that BnaFAX1-1 may contribute to the formation of seed high oil content in B. napus. Furthermore, to further determine whether BnaFAX1-1 and BnaFAX1-2 are conducive to the formation of high biological yield (Additional file 1: Figure S1), the seedling leaves of four pairs with extremely high- (P281, P542, P125, P257-HBY) and low-biological yield accessions (P319, P276, P131, P81-LBY) were selected for qRT-PCR analysis, and biological yield dry weight per plant for each accession is shown in Additional file 1: Figure S3G. The qRT-PCR results showed that the expression levels of BnaFAX1-1 and BnaFAX1-2 in high-biological yield accessions were higher than those in low-biological yield accessions (Additional file 1: Figure S3E, F), which is consistent with the GWAS analysis result (Additional file 1: Figure S1). To further determine whether BnaFAX1-1 can increase both seed oil content and biological yield, we characterized the function of BnaFAX1-1.

\section{Subcellular localization and transcript levels of BnaFAX1-1 in B. napus}

To determine the subcellular localization of BnaFAX1-1 in plant cells, we tagged BnaFAX1-1 with green fluorescent protein (GFP) and expressed the construct under the control of the constitutive Cauliflower mosaic virus (CaMV) 35S promoter (Fig. 2A). We transiently transfected Arabidopsis protoplasts with the BnaFAX1-1-GFP construct, using AtFAX1-GFP as a marker for chloroplast envelopes. We observed a ring of fluorescence at the periphery of chloroplasts, which is consistent with a plastid envelope localization for BnFAX1-1, as seen previously with AtFAX1 (Fig. 2B).

We next measured BnaFAX1-1 transcript levels of in seven tissues across five stages of development (roots, stems, leaves, flowers, buds, seeds, and silique pericarp after flowering at 7, 14, 21, 30 and 40 days). We observed the highest expression level for BnaFAX1-1 in leaves and seeds after 40 days of flowering (Fig. 2C). We had previously determined that AtFAX1 was mainly expressed in leaves, but not in seeds [21]. This result suggested that BnaFAX1-1 function may differ from that of AtFAX1, which did not contribute to seed oil accumulation in Arabidopsis.

\section{Overexpression of BnaFAX1-1 in Arabidopsis and B. napus}

Next, we generated BnaFAX1-1 overexpression constructs and transformed both Arabidopsis (Col-0 accession) and $B$. napus (Westar cultivar). We validated overexpression lines (OE) by RT-qPCR using total RNA extracted from B. napus and Arabidopsis transgenic individuals with BnaFAX1-1-specific primers (Additional file 1: Table S4). We selected four homozygous BnaFAX1-1 overexpressing lines in Arabidopsis, named OE/ At\#1, OE/At\#2, OE/At\#3, and OE/At\#4 (Fig. 3A). Similarly, we obtained four BnaFAX1-1 overexpressing lines in B. napus, named OE\#17, OE\#19, OE\#20 and OE\#21 (Fig. 4A).

\section{Increased biological yield and seed oil production by BnaFAX1-1 overexpression in Arabidopsis}

Next, we analyzed the phenotype of Arabidopsis lines overexpressing BnaFAX1-1 and compared their growth to wild-type (WT) plants. We noticed that all Arabidopsis BnaFAX1-1 overexpressing lines were slightly larger and produced more biomass than WT plants (Fig. 3B, Table 1). After reaching reproductive maturity, overexpression lines were significantly bigger than the WT, with thicker inflorescence stalks and more siliques (Fig. 3C, Table 1). A detailed analysis of different tissues and organs 


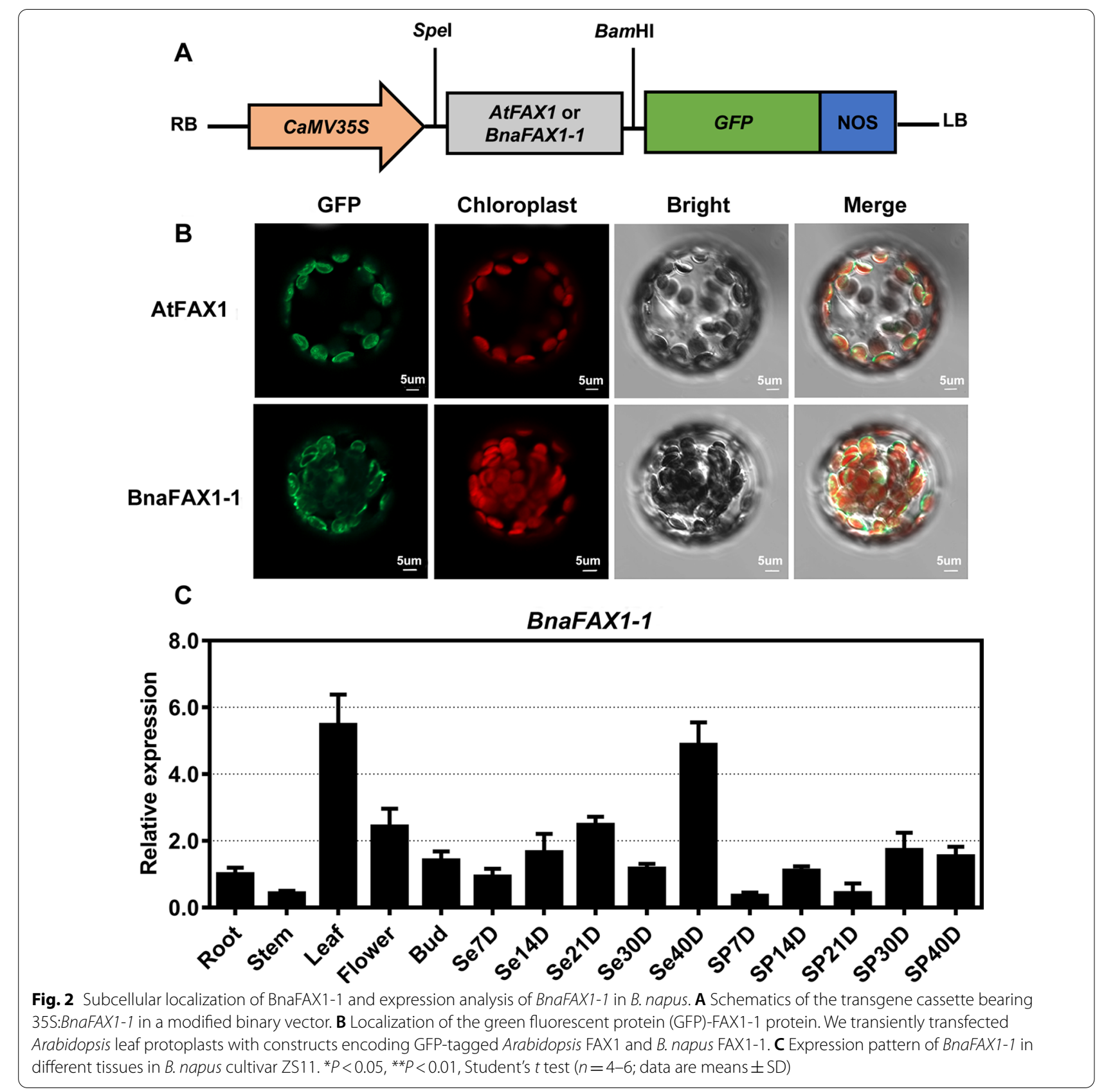

in transgenic and WT plants grown for 7 weeks revealed that plant height, rosette fresh and dry weight, fresh and the dry weight of biological yield was significantly higher in overexpressing lines relative to WT plants (Table 1). Likewise, stem fresh weight and stem diameter in the transgenic lines were significantly increased compared to WT plants. Furthermore, we observed an increase in seed yield per plant in overexpression lines, largely due to an increase in silique number per plant (Table 1). We also determined the total lipid content of mature seeds, which indicated that overexpression lines accumulated more total lipid content relative to the WT (Fig. 3D). Collectively, these results indicated that the heterologous overexpression of BnaFAX1-1 in Arabidopsis promoted plant growth and development, and led to an increase in seed oil production. 

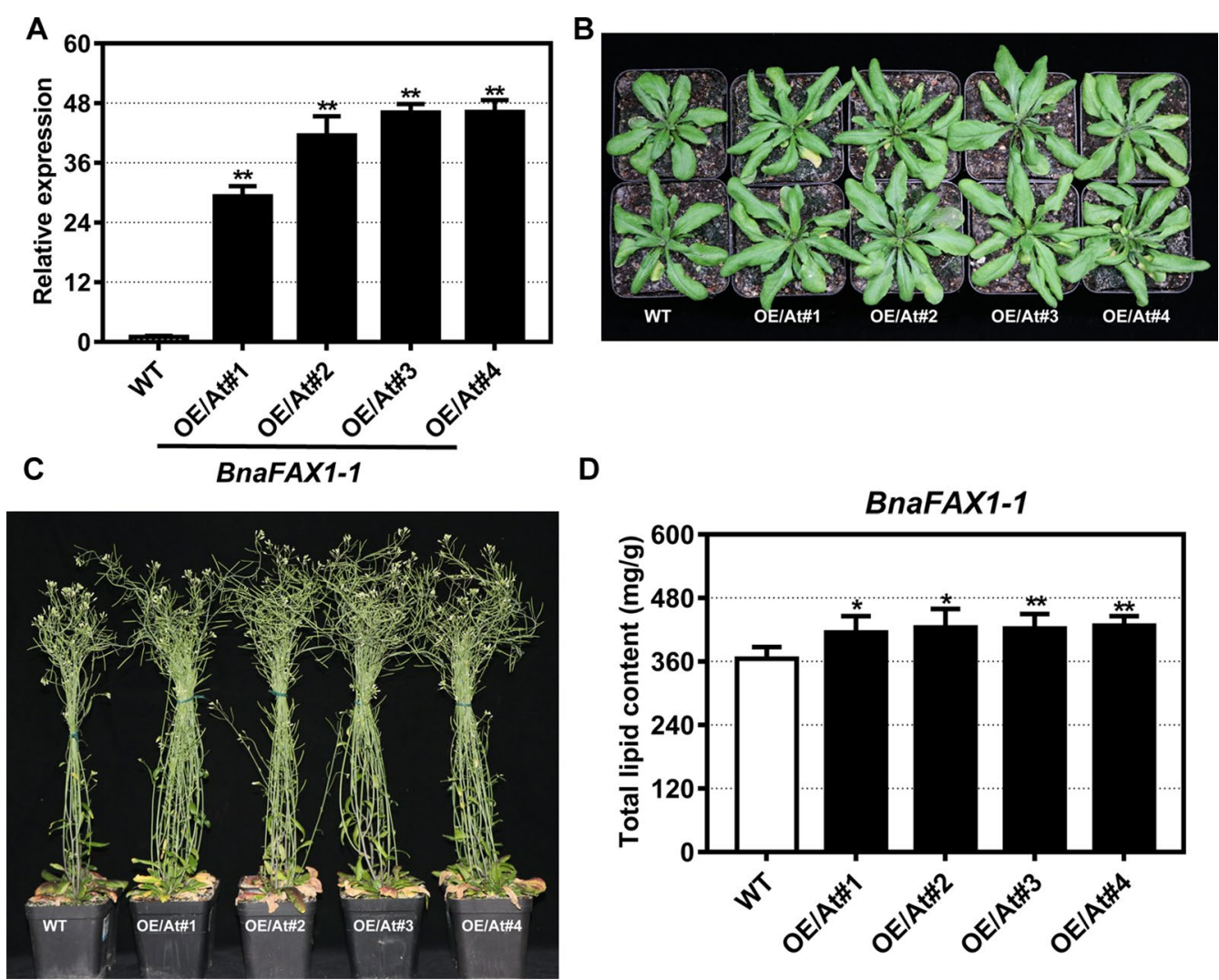

Fig. 3 Phenotypic analysis of Arabidopsis lines overexpressing BnaFAX1-1. A Relative expression levels of BnaFAX1-1 in Arabidopsis overexpressing lines. B, C Growth characteristics of 30-day-old (B) and 49-day-old plants (C) grown in incubators. D Total lipid content and fatty acid composition in mature Arabidopsis seeds. ${ }^{*} P<0.05,{ }^{*} P<0.01$, Student's $t$ test $(n=4-6 \pm$ SD)

\section{Increased biological yield, gibberellin and leaf lipid} contents in B. napus plants overexpressing BnaFAX1-1

To test the effect of BnaFAX1-1 overexpression in $B$. napus on biomass accumulation, we analyzed the growth kinetics of three independent BnaFAX1-1 overexpression lines selected at random (OE\#17, OE\#19 and OE\#21) (Fig. 4A). We grew all plants hydroponically in Hoagland nutrient solution for 32 days. All BnaFAX1-1 overexpression lines were larger and produced more biomass than their non-transgenic WT control (Fig. 4B). This increase in leaf biomass was reflected in all phenotypes measured: leaf fresh/dry weight and leaf size (including leaf length, leaf width and leaf area; Fig. 4C, D). Compared to the WT, overexpression of BnFAX1-1 also resulted in a significant increase in total root length, root area, root volume, root fresh and dry weight (Fig. 4D). Overexpression of BnaFAX1-1 in B. napus, therefore, promoted plant growth.

Toward the identification of the potential mechanism linking FAX1 and B. napus growth and biomass improvements, we quantified phytohormone contents in the leaves of two transgenic lines (OE\#19 and OE\#21) and their WT using liquid chromatography followed by tandem mass spectrometry (LC-MS/MS). We observed that gibberellic acid A4 (GA4) accumulated to significantly higher levels in transgenic leaves relative to the WT (Fig. 5A). By contrast, the contents of indole-3-acetic acid (IAA), salicylic acid (SA), and jasmonic acid (JA) were similar in the overexpression lines and the WT (Additional file 1: Figure S4). GA4 is a bioactive gibberellin that plays critical roles in plant growth and development [11]. To explore the reason behind the increase in GA4 content in the transgenic lines, we performed transcriptome sequencing from leaves of the transgenic lines (OE\#19 and OE\#21) and WT. We discovered that the GA4 biosynthetic genes COPALYL DIPHOSPHATE SYNTHASE (CPS), KAURENOIC ACID OXIDASES (KAOS) and GA20 OXIDASE (GA20OX) were more highly expressed in the transgenic lines relative to the WT (Fig. 5B). We validated these results by RT-qPCR (Fig. 5C). These results indicate that overexpression of BnaFAX1-1 led to up-regulated GA4 biosynthesis, which may in turn contribute to biological yield increase in $B$. napus. 


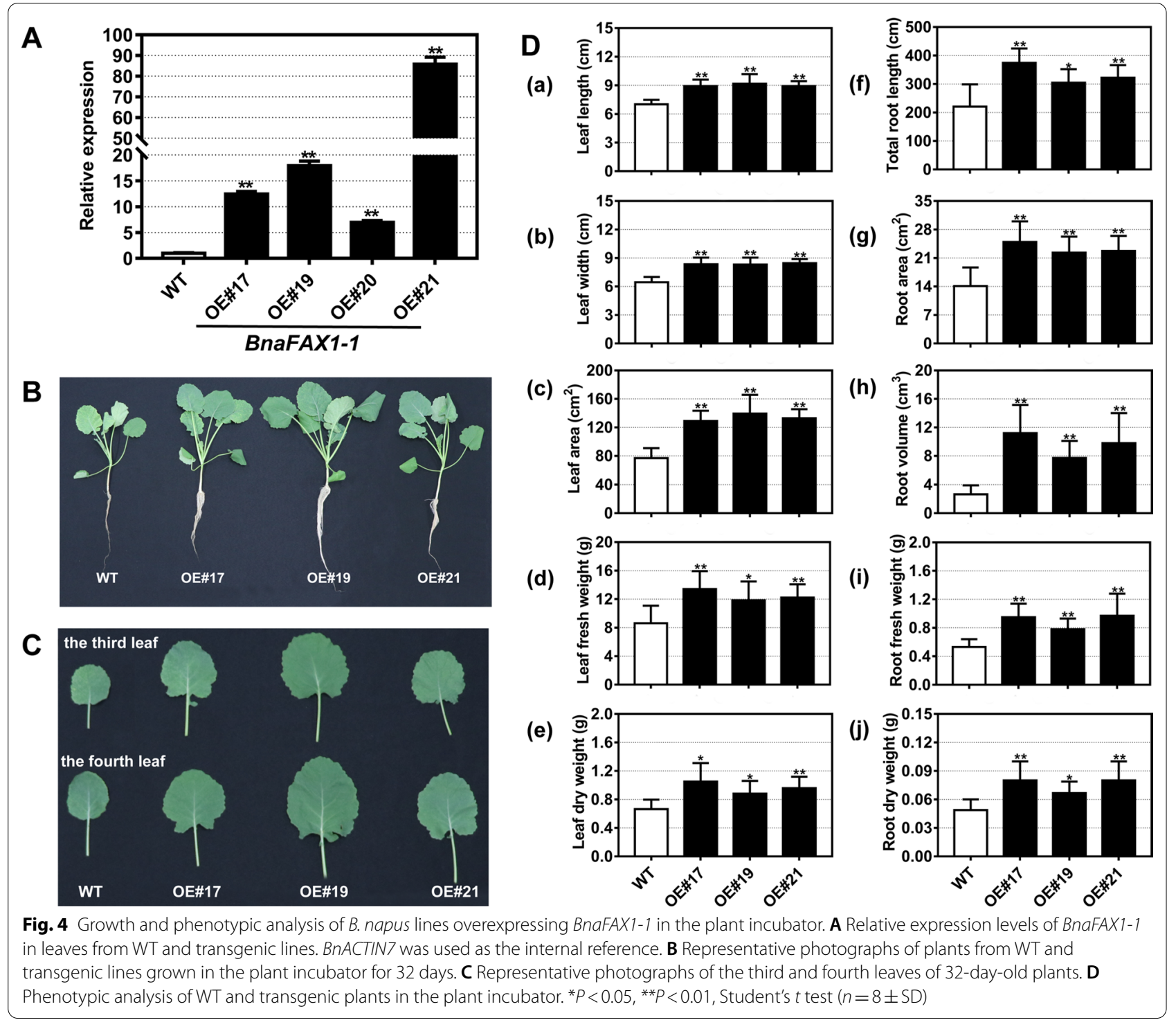

To investigate the consequence of BnaFAX1-1 accumulation in the two selected overexpression lines above on membrane lipid contents, we analyzed lipids from 32-day-old leaves using LC-MS/MS (Fig. 6). We observed a higher lipid content for phosphatidylcholine (PC) and phosphatidylethanolamine (PE) in the leaves of OE\#19 and OE\#21 plants when compared to WT (Fig. 6). These results revealed that BnaFAX1-1 share the same role as AtFAX1 when ectopically expressed in leaves for the regulation of leaf lipid and biomass accumulation.

\section{BnaFAX1-1 enhances biological yield and seed yield of $B$. napus plants grown in the field}

To determine if the phenotypes seen in BnaFAX1-1 overexpression lines extended to the field, we sowed seeds for WT and OE lines in a randomized field plot design using four plots for each OE line and WT. We investigated growth characteristics in plants at the flowering stage (grown for 175 days). OE plants were clearly bigger and taller compared to $\mathrm{WT}$, and produced more leaves on the main stem in the same growth period (Fig. 7A, C). In addition, OE plants showed larger leaves at the same position relative to $\mathrm{WT}$ plants, which was reflected in increased leaf length, leaf width and leaf area (Fig. 7B, C). Finally, OE lines produced thicker main stems than WT plants; only chlorophyll content and the photosynthetic rate of OE lines were similar to those of the WT (Fig. 7C).

We next harvested mature plants from the field to carry out additional measurements. The BnaFAX1-1 OE lines were significantly taller than WT plants and bore 
Table 1 Plant biomass and seed yield in Arabidopsis Col-0 plants and Arabidopsis lines overexpressing BnaFAX1-1

\begin{tabular}{llcccc}
\hline Traits & WT & OE/At\#1 & OE/At\#2 & OE/At\#3 & OE/At\#4 \\
\hline Stem fresh weight (mg/cm) & $12.57 \pm 0.79$ & $12.63 \pm 2.03$ & $15.31 \pm 2.00^{* *}$ & $15.9 \pm 1.28^{* *}$ & $15.86 \pm 2.66^{* *}$ \\
Stem diameter (mm) & $0.97 \pm 0.11$ & $1.04 \pm 0.08$ & $1.16 \pm 0.99^{* *}$ & $1.23 \pm 0.14^{* *}$ & $1.21 \pm 0.08^{* *}$ \\
Plant height (cm) & $32.6 \pm 3.37$ & $39.74 \pm 2.21^{* *}$ & $38.9 \pm 1.13^{* *}$ & $37.75 \pm 2.5^{* *}$ & $37.49 \pm 2.37^{* *}$ \\
Rosette fresh weight (g) & $1.09 \pm 0.14$ & $1.28 \pm 0.17^{*}$ & $1.43 \pm 0.3^{*}$ & $1.58 \pm 0.36^{* *}$ & $1.44 \pm 0.32^{*}$ \\
Rosette dry weight (g) & $0.17 \pm 0.02$ & $0.18 \pm 0.02$ & $0.22 \pm 0.02^{* *}$ & $0.23 \pm 0.05^{* *}$ & $0.23 \pm 0.04^{* *}$ \\
Fresh weight of BYAG (g) & $2.91 \pm 0.24$ & $4.44 \pm 0.48^{* *}$ & $4.20 \pm 0.45^{* *}$ & $4.85 \pm 0.57^{* *}$ & $4.31 \pm 0.43^{* *}$ \\
Dry weight of BYAG (g) & $0.45 \pm 0.05$ & $0.65 \pm 0.07^{* *}$ & $0.68 \pm 0.04^{* *}$ & $0.76 \pm 0.10^{* *}$ & $0.65 \pm 0.14^{* *}$ \\
Length of siliques (cm) & $1.6 \pm 0.09$ & $1.57 \pm 0.04$ & $1.62 \pm 0.09$ & $1.67 \pm 0.07$ & $1.59 \pm 0.1$ \\
Number of siliques per plant & $349.7 \pm 24.18$ & $408.7 \pm 32.1^{* *}$ & $416.4 \pm 39.05^{* *}$ & $501.9 \pm 45.95^{* *}$ & $412.75 \pm 57.54^{*}$ \\
Number of seeds per silique & $61.06 \pm 6.24$ & $64.33 \pm 3.56$ & $60.78 \pm 4.93$ & $63.33 \pm 5.69$ & $61.94 \pm 6.63$ \\
Weight of per 1000 seeds (mg) & $19.15 \pm 0.28$ & $18.37 \pm 0.68$ & $18.58 \pm 0.11$ & $19.12 \pm 0.12$ & $19.36 \pm 0.58$ \\
Seed yield per plant (mg) & $118.5 \pm 34$ & $179.8 \pm 45^{* *}$ & $174.9 \pm 54^{*}$ & $241.7 \pm 51^{* *}$ & $172.0 \pm 42^{*}$ \\
\hline
\end{tabular}

BYAG: biological yield of above ground organs

${ }^{*} P<0.05,{ }^{* *} P<0.01$, Student's $t$ test $(n=6-10 \pm$ SD)
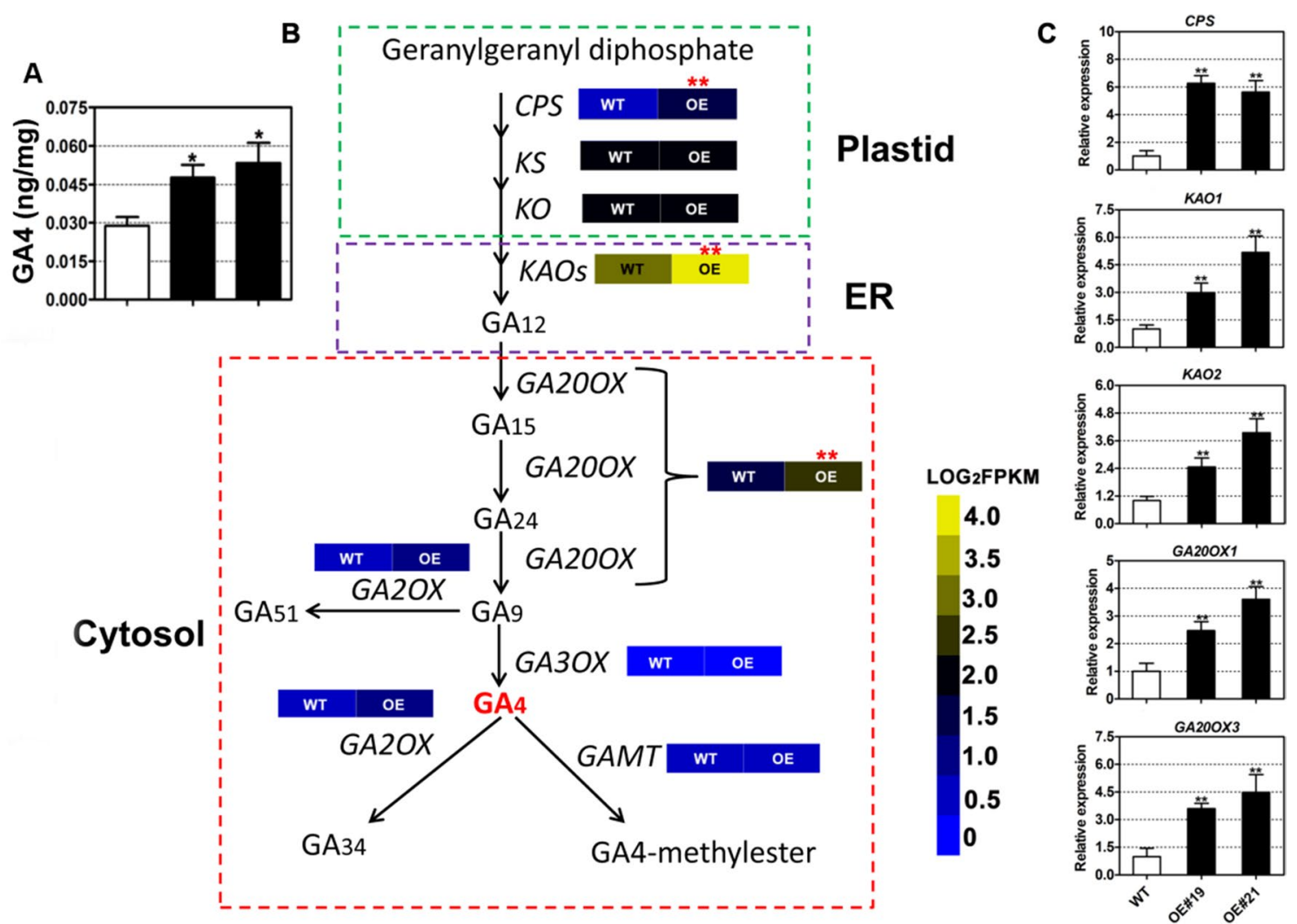

Fig. 5 Overexpression of BnaFAX1-1 in B. napus upregulates gibberellic acid biosynthesis, which may contribute to biological yield increase. A GA4 (gibberellic acid A4) content in leaves from 32-day-old WT and B. napus lines overexpressing BnaFAX1-1. B Expression patterns of GA4 biosynthetic and metabolic genes. The yellow indicates higher gene expression levels and the blue indicates lower gene expression levels. COPALYL DIPHOSPHATE SYNTHASE, CPS; KAURENE SYNTHASE, KS; KAURENE OXIDASE, KO; KAURENOIC ACID OXIDASES, KAOS; GA 20-OXIDASE, GA20OX; GA 2-OXIDASE, GA2OX; GA 3-OXIDASE, GA3OX; GIBBERELLIC ACID CARBOXYL METHYLTRANSFERASE, GAMT. C Confirmation of the relative expression levels of selected genes by RT-qPCR. ${ }^{*} P<0.05,{ }^{*} P<0.01$, Student's $t$ test $(n=4-6 \pm$ SD) 


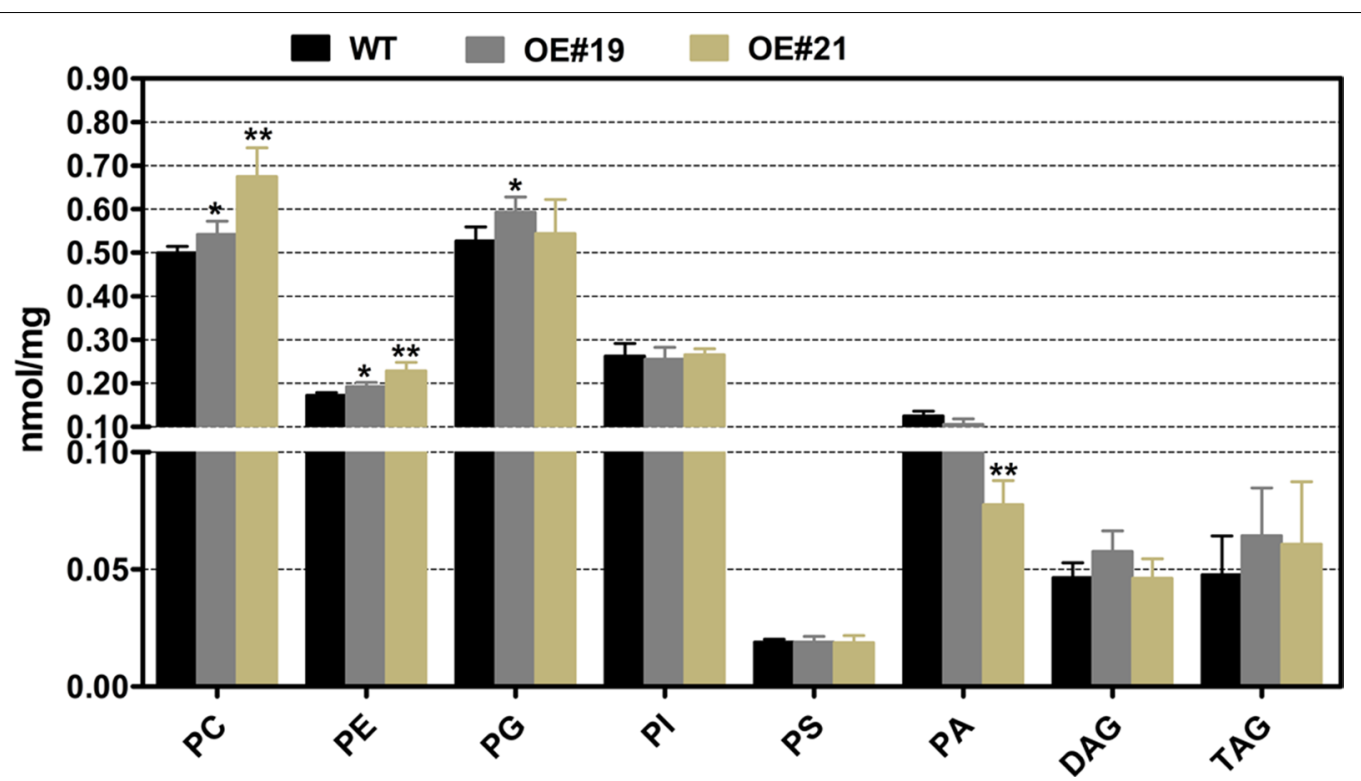

Fig. 6 Accumulation of major polar membrane lipids species in leaves from 32-day-old WT and lines overexpressing BnaFAX1-1. phosphatidylcholine, PC; phosphatidylethanolamine, PE; phosphatidylglycerol, PG; phosphatidylinositol, PI; phosphatidylserine, PS; phasphatidic acid, PA; diacylglycerol, DAG; triacylglycerol, TAG. ${ }^{*} P<0.05,{ }^{*} P<0.01$, Student's $t$ test $(n=4-6 \pm$ SD)

more effective branches (i.e., branches bearing seeds) per plant (Fig. 8A, D-c). Although we observed no differences in the length of the main inflorescence between the WT and transgenic plants, the OE lines did exhibit more siliques per main inflorescence than in WT plants (Fig. 8B). Similarly, total silique number was significantly greater in all OE lines relative to WT (Fig. 8D-f), as were silique length (Fig. 8C) and number of seeds per silique. Together, these results revealed the greater seed yield per plant and biological yield in all OE plants (Fig. 8D), possibly by increasing the number of effective branches and siliques per plant. We did not observe such phenotypes when we overexpressed AtFAX1 in Arabidopsis in our previous work [21]. Therefore, BnaFAX1-1, unlike AtFAX1, may play a vital role in improving seed yield and biological yield in B. napus.

\section{BnaFAX1-1 enhances $B$. napus seed oil production and improves oil quality}

We examined total lipid content in seeds from OE lines and WT at $30 \mathrm{~d}$ and $45 \mathrm{~d}$ after flowering, as well as in dry seeds following harvest. Overexpression of BnFAX1-1 in $B$. napus resulted in an increase in total seed lipid contents at all developmental stages tested relative to WT (Fig. 9A). We also determined the range of triacylglycerol (TAG) molecular species and total TAG content in BnaFAX1-1 OE lines and WT mature dry seeds. We saw a significant rise in the content of many TAG molecular species (TAG 50:2, 50:3, 52:2, 54:2, 54:3, 54:4, 54:5, 56:2,
56:4) and total TAG content in BnaFAX1-1 OE transgenic plants compared to WT (Fig. 9B, C). In addition, an analysis of fatty acid composition of mature dry seeds grown in the field revealed that oleic acid (C18:1) was significantly increased in the transgenic lines, whereas palmitic acid (C16:0), arachidic acid (C20:0) and eicosenic cis (C20:1) were significantly reduced. Stearic acid (C18:0), linoleic acid (C18:2) and linolenic acid (C18:3) contents were similar in the OE lines and WT (Fig. 9D). These results demonstrated that overexpression of $\mathrm{Bna}$ FAX1-1 effectively increased seed oil production and oleic acid content. These results, therefore, revealed that BnaFAX1-1 may have important application value in $B$. napus molecular breeding to improve seed oil content, oil quality, seed yield and biological yield.

\section{Discussion}

As the third largest source of plant oil after oil palm (Elaeis guineensis) and soybean (Glycine max), rapeseed (Brassica napus) produces approximately 15\% of edible vegetable oil worldwide [13, 27, 31]. Enhancing both seed oil production and seed oil quality is a major goal in $B$. napus breeding, which can be accomplished by improving seed yield, seed oil content and biological yield, separately or in combination. The present study provides a possible and simple solution to simultaneously improve seed oil production, oil quality, seed yield, seed oil content and biological yield in $B$. napus by raising the expression levels of a single gene. We identified the potential 
A

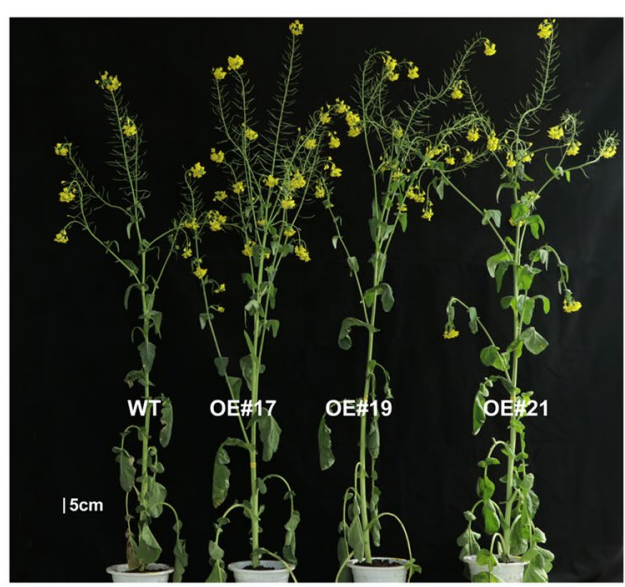

B

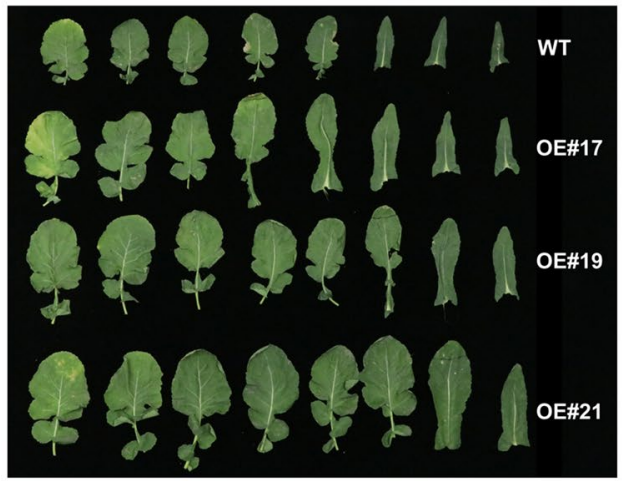

C

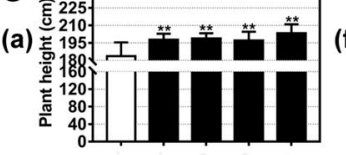

(b)

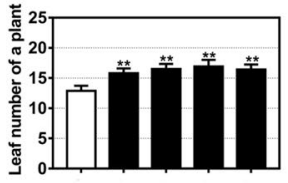

(c)

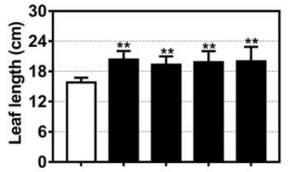

(d)

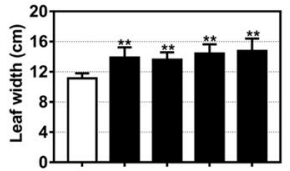

(e)

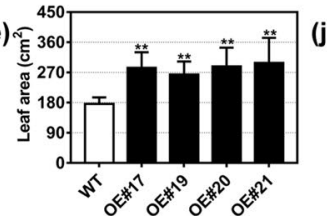

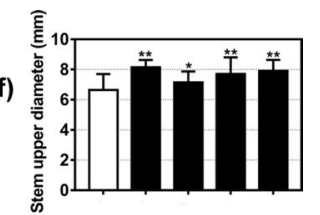

(g)

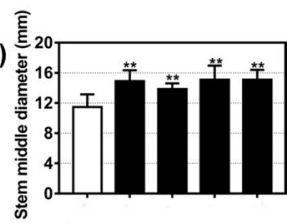

(h)
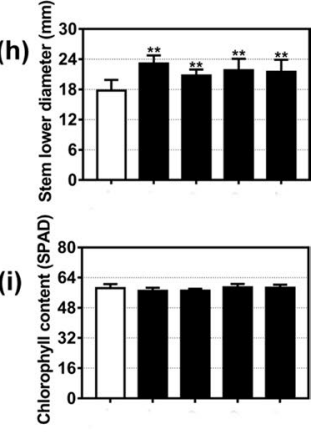

(j)

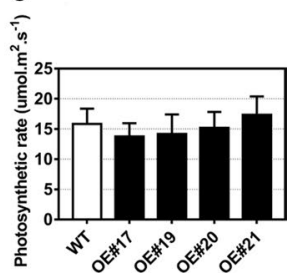

Fig. 7 Growth of B. napus lines overexpressing BnaFAX1-1 in the field. A Representative photographs of 175-day-old plants grown in the field. B Representative photographs of leaves at the same position. C Summary of phenotypic analysis of WT and B. napus lines overexpressing BnaFAX1-1 in the field. Plant height, a; plant leaf number, b; leaf length, c; leaf width, d; leaf area, e; upper stem diameter, f; middle stem diameter, g; lower stem diameter, h; chlorophyll content i; photosynthetic rate, j. ${ }^{*} P<0.05,{ }^{*} P<0.01$, Student's $t$ test $(n=10 \pm$ SD)

key gene BnaFAX1-1 through the identification of putative chloroplast-localized transporters whose encoding genes mapped closely to significant SNPs from a previous GWAS of 520 B. napus accessions for biological yield. Increasing expression of BnaFAX1-1 improved biological yield and seed oil production, especially oleic acid content, which has not been observed in previous studies.

Chloroplasts are the main site of photosynthesis and plant fatty acid production. Recent studies revealed that altering carbon metabolism in the chloroplasts of transgenic oil crops improved biological yield and seed oil production. We hypothesized that export of photosynthates from the chloroplast may contribute to improving biological yield and seed oil production. We, therefore, focused here on genes encoding proteins predicted to locate to the chloroplast membrane, leading to a first selection of 29 candidate genes (Additional file 1: Table S2). From further analysis of these 29 genes, we identified a gene encoding a putative transporter: BnaA07g17240D
(BnaFAX1-1). The Arabidopsis ortholog of BnaFAX1-1 is the putative fatty acid exporter AtFAX1, which is crucial for biological yield. Both biological yield and seed oil production increased in 35S:BnaFAX1-1 transgenic $B$. napus plants grown in the field relative to WT plants, highlighting the potential of this gene to improve seed oil production in B. napus (Figs. 4, 6, 7, 8, 9), which the positive effect of BnaFAX1-1-OE in Brassica napus is generally consistent with prior reports on AtFAX1 gene being overexpressed individually in Arabidopsis [41] and camelina sativa [4]. A new finding of the present study is that overexpression of BnaFAX1-1 did not affect seed weight in Brassica napus, while the plant biomass and seed oil yield were increased dramatically when BnaFAX1-1 was overexpressed in rapeseed.

Furthermore, we determined that the expression of GA biosynthetic genes was up-regulated, in agreement with the higher GA4 levels measured in the OE lines (Fig. 5). GA biosynthesis is derived from geranylgeranyl 

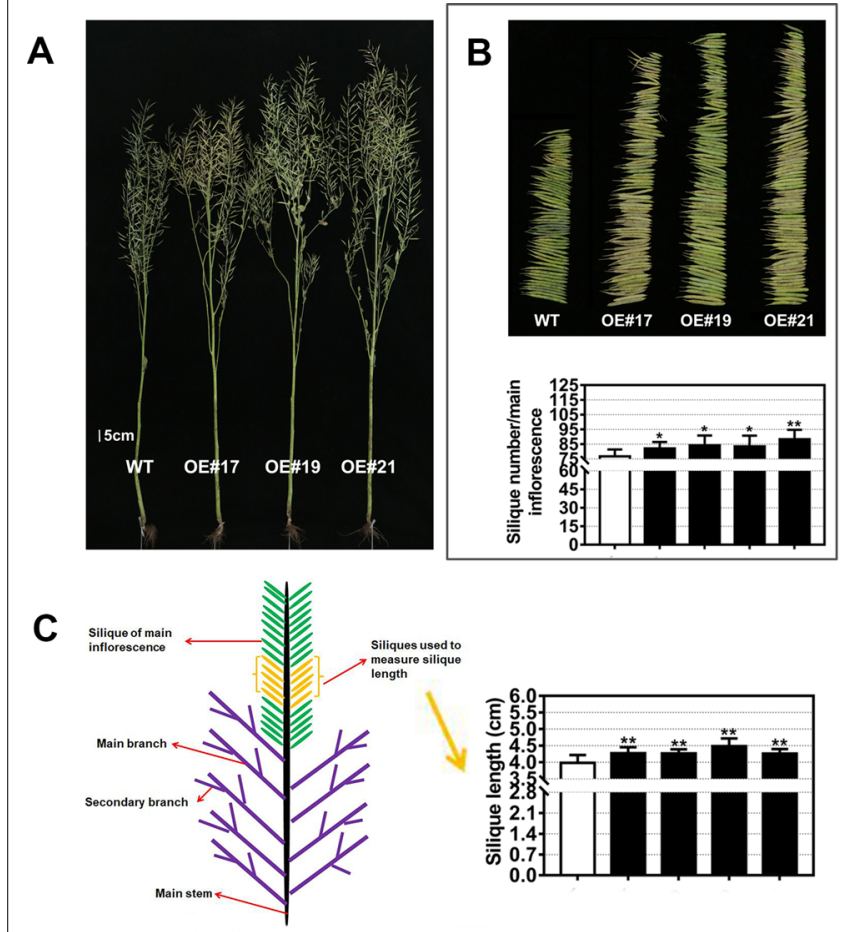

D

(a)

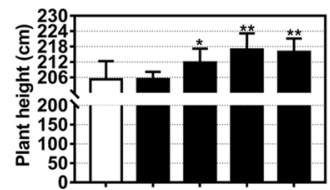

(b) $\stackrel{8}{8}$

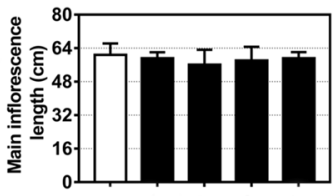

(c)

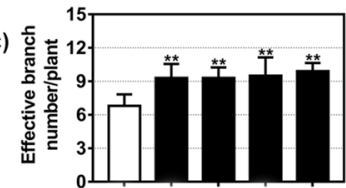

(d)

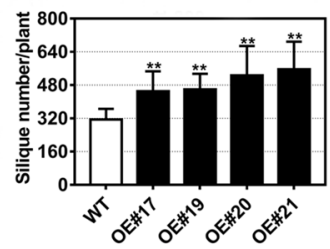

(e)
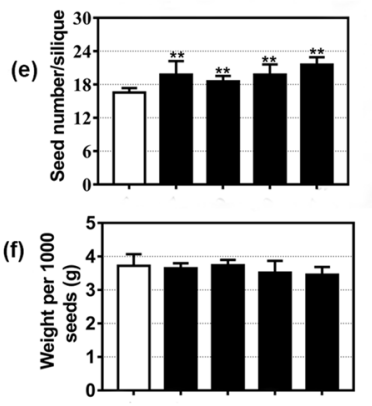

(g)

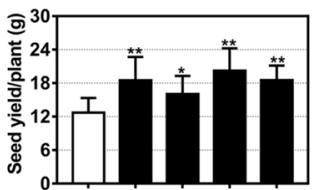

Fig. 8 Phenotypes of B. napus lines overexpressing BnaFAX1-1 after harvest in the field. A Representative photographs of mature plant at harvest. B Silique per main inflorescence from mature plants at harvest and statistic of the silique number per main inflorescence. $\mathbf{C}$ Simplified diagram of Brassica napus and statistic of silique length. D Summary of phenotypic analysis of B. napus WT and lines overexpressing BnaFAX1-1 in the field. Plant height, a; main inflorescence length, b; effective branch number (seed-bearing), c; silique number per plant, d; seed number per silique, e; weight per 1000 seeds, f; seed yield per plant, g; biological yield above ground, $h .{ }^{*} P<0.05,{ }^{*} P<0.01$, Student's $t$ test $(n=10 \pm$ SD)

diphosphate (GGDP), which is synthesized in the chloroplast. GGDP is then converted to ent-kaurene by ent-COPALYL DIPHOSPHATE SYNTHASE (CPS) and ent-KAURENE SYNTHASE (KS). Ent-kaurene is then converted to ent-kaurenoic acid, which is catalyzed by the plastid envelope-located ent-kaurene oxidase (AtKO1) [16, 47]. ent-kaurene acid is then exported from the chloroplast to complete GA biosynthesis on the endoplasmic reticulum (ER). Fatty acid export may affect carbon metabolism in plastids and further affect ent-kaurene contents or export from plastids. It's possible that BnaFAX1 may affect the carbon-hydrate types in plastids, which further affected contents of GA precursors in plastids, such as GGDP, and related enzymes contents, such as CPS. No GA precursor exporter from plastid has been reported [15]. As AtFAX1 was reported as a plastid inner-envelope localized exporter, it's possible that BnaFAX1-1 may also localized at inner-envelope of plastids and may export fatty acids and also ent-kaurene etc. It would be interesting to investigate whether BnaFAX1 may be involved in GA precursor export from plastid. GA biosynthesis was reported to improve plant biological yield [11]. BnaFAX1-1 may indirectly affect GA biosynthesis, resulting in the observed increase in biological yield and seed yield, as seen in BnaFAX1-1 overexpression lines.

Increasing seed yield and seed oil content are two major approaches to enhance seed oil production. In B. napus, seed yield is largely determined by three yield component traits: silique number per plant, seed number per silique, and the weight per thousand seeds [29]. In the present study, the increase in seed yield measured for B. napus BnaFAX1-1-OE lines was mainly due to silique number per plant and seed number per silique, as we detected no differences in the weight per thousand seeds between BnaFAX1-1-OE and WT plants (Fig. 8C). Seed oil content is another essential contributor to seed oil production, which constitutes the basis for $B$. napus economic importance. Overexpressing BnaFAX1-1 in Arabidopsis and $B$. napus led to a significant rise in seed oil content relative to WT (Figs. 3D, 9A-C). Therefore, overexpressing BnaFAX1-1 has great potential to increase seed oil production in B. napus.

In our previous study, Arabidopsis lines overexpressing FAX1 showed a pronounced increase of lipids in flowers and leaves [21]. However, the $f a x 1$ loss of 


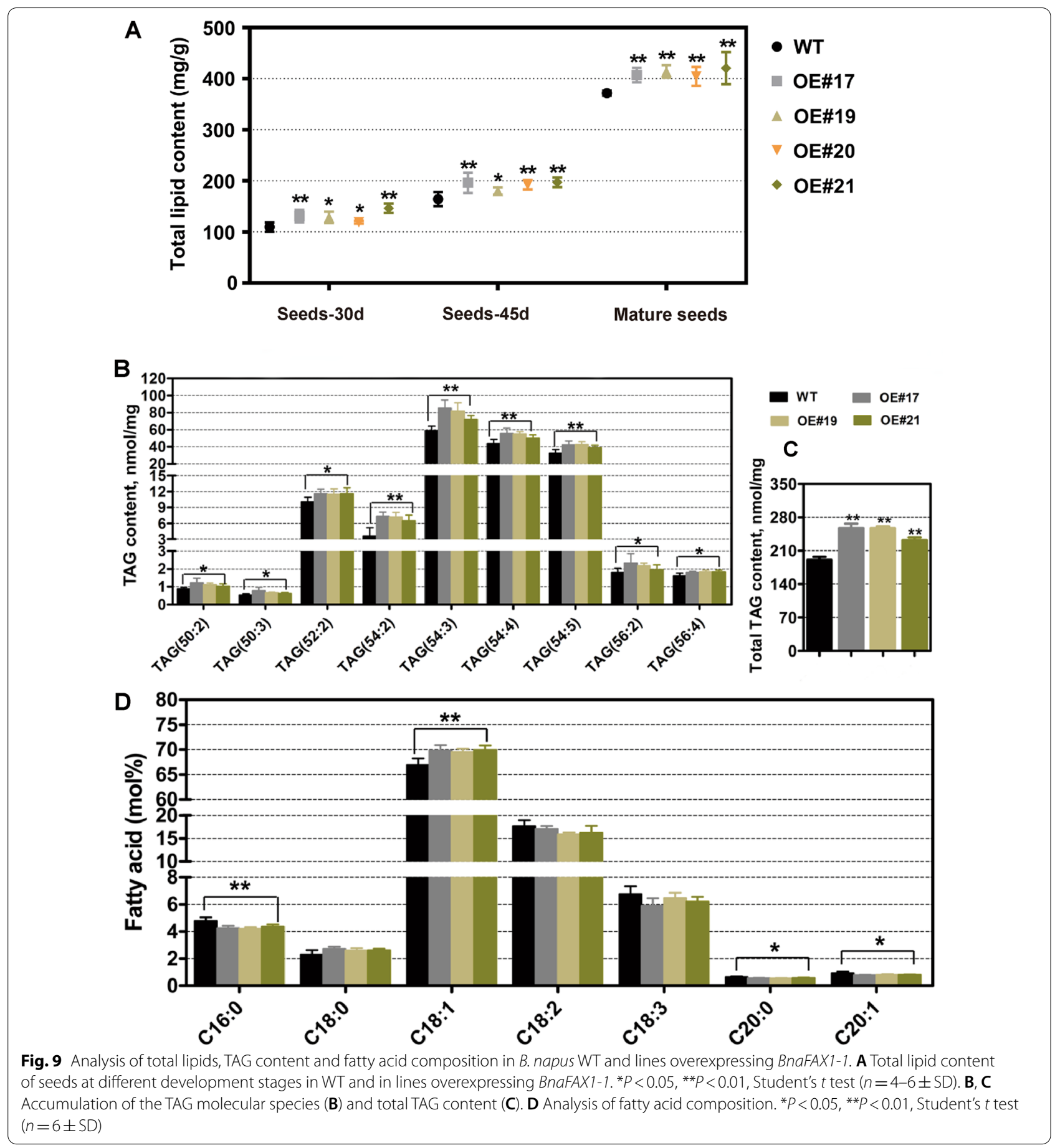

function mutation had no effects on seed oil content or seed yield [21, 23]. In the green alga Chlamydomonas (Chlamydomonas reinhardtii), the overexpression of either CrFAX1 or CrFAX2 resulted in the accumulation of intracellular TAG [24]. Recently, AtFAX2 and AtFAX4 were reported to be seed-specific transporters mediating seed embryo fatty acid export for seed oil content accumulation in Arabidopsis [22]. These results reveal that the tissue-specificity of $F A X$ expression may contribute to lipid accumulation in specific tissues. Notably, we determined that BnaFAX1-1 is highly expressed in siliques during the seed-filling stage in a cultivar with high seed oil content, when compared to 
another cultivar with low seed oil content, indicating a correlation between BnaFAX1-1 function and the regulation of seed oil content and seed yield (Fig. 1B). The present study reveals that BnaFAX1-1 mainly mediates seed plastid fatty acid export for the accumulation of seed TAG during the seed-filling stage, leading to the measured increase in seed oil content in the overexpression lines. By contrast, AtFAX1 had no effect on seed TAG accumulation in Arabidopsis, as AtFAX1 is expressed at low levels during the seed-filling stage.

Oleic acid (C18:1) is an important unsaturated fatty acid component of $B$. napus oil with high nutritional value and good thermal stability. Oil with high oleic acid content can reduce the risk of cardiovascular disease in overweight individuals [38] and effectively prevent arteriosclerosis [35]. In addition, it is highly resistant to oxidization and degradation at high temperature and enjoys a long shelf life [39]. Such oleic acid-rich oils also emit little to no smoke when heated to high temperatures and reduce cooking times [34], display high stability during frying, and imbue frying products with excellent aroma. Aside from cooking applications, oleic acid is also a raw material for biodiesel production [37]. In the present study, we determined that oleic acid (C18:1) content significantly increased in BnaFAX1-1-OE seeds, while another plastid-derived fatty acid palmitic acid (16:0) decreased relative to WT (Fig. 9C). In our previous study, AtFAX1 affected the content of plastid-derived fatty acids in Arabidopsis leaves and flowers, but not in seeds [21]. AtFAX2 and AtFAX4 mainly mediate oleic acid (C18:1) and palmitic acid (16:0), as these two fatty acids are the major free fatty acid form in plastids [22]. However, the fatty acids composition of TAG in mature seeds of $f a x 2, f a x 4, f a x 2 f a x 4$ and wild types shows no obvious difference, which indicates that neither FAX2 nor FAX4 affects the relative probability of fatty acid composition in TAG of seeds. Arabidopsis ATP-BINDING CASSETTE A9 (ABCA9) mediated fatty acids and acyl-CoA import into the endoplasmic reticulum for TAG accumulation. However, the fatty acid composition and contents were not affected by a loss of function in AtABCA9 [18]. Our results suggest that BnaFAX1-1 may selectively mediate the export of specific plastid-derived fatty acids, such as oleic acid, and the potential mechanism would be the subject of future work.

In conclusion, we identified a fatty acid export protein, BnaFAX1-1, which mediates fatty acid export from plastids in developing seeds. The overexpression of BnaFAX1-1 significantly increased seed oil content, oil quality, seed yield and biological yield in B. napus. Bna$F A X 1-1$ overexpression lines resulted in an up-regulation of GA4 biosynthesis, indicating that BnaFAX1-1 overexpression may influence GA biosynthesis, leading to increased biological yield and seed oil production. Furthermore, BnaFAX1-1 contributed to the accumulation of oleic acid, an unsaturated fatty acid of high economic value, in seeds, thus improving oil quality. The present study provides an important solution to simultaneously improve rapeseed seed oil production, seed oil content, seed yield, biological yield and seed oil quality by modulating the expression of BnaFAX1-1. We propose that BnaFAX1-1 should be a potential target for B. napus molecular breeding in the future.

\section{Experimental procedures}

\section{Phylogenetic analyses, protein properties and sequence} analyses of the FAX family

We downloaded the sequences of FAX proteins from Arabidopsis (Arabidopsis thaliana), field mustard (Brassica rapa), wild cabbage (Brassica oleracea), and B. napus to generate on-multiple protein sequence alignments using the integrated MUSCLE program in MEGA7.0 [19]. We also generated a phylogenetic tree of the FAX family with MEGA7.0 software using the Neighbor-Joining (NJ) method and bootstrap analysis (1000 replications). We visualized the phylogenetic trees in FigTree v1.4.2 [1]. We deduced the physical properties of BnaFAX proteins (molecular weight [MW] and isoelectric point [pI]) using the online proteomics database ExPASy (http://web. expasy.org/peptide_mass/) [12]. We predicted transmembrane domains using the online tool TMHMM Server v. 2.0 (http://www.cbs.dtu.dk/services/TMHMM/) [6]. We determined the chromosomal location of all BnaFAX genes by performing Basic Local Alignment Search Tool for Nucleotides (BLASTN) searches of their sequences against the 19 B. napus chromosomes, followed by visualization with the MapChart Software [43]. We identified exon/intron boundaries for the BnaFAX genes at the Gene Structure Display Server (GSDS 2.0, http://gsds.cbi. pku.edu.cn/index.php) [14] and conserved protein motifs with the MEME suite of programs (http://meme-suite. $\operatorname{org} /$ ) [3], respectively. We then annotated all identified conserved motifs with InterProScan (http://www.ebi.ac. $\mathrm{uk} /$ Tools/pfa/iprscan/) according to MAST data from the MEME program [17].

\section{Vector constructs and plant transformation}

We PCR-amplified the complete coding sequence (CDS) of the BnaFAX1-1 gene from B. napus cDNA using the primer pair BnaFAX1-1 FP(XbaI) + BnaFAX1-1 RP(SacI) (primers used in this study are listed in Additional file 1: Table S4). We then subjected the PCR product to restriction digest with $\mathrm{XbaI}$ and $S a c \mathrm{I}$ and ligated the purified digested product into the pCAMBIA2301M vector (a modified version of pCAMBIA2301 available in our laboratory) to generate pCAMBIA2301-35S:BnaFAX1-1. We 
introduced the pCAMBIA2301-35S:BnaFAX1-1 construct into Agrobacterium (Agrobacterium tumefaciens) strain GV3101 for transformation of Arabidopsis Col-0 accession by the floral dip method [5]. We selected positive transgenic by germinating seeds on Murashige and Skoog (MS) medium supplemented with $10 \mathrm{mg} / \mathrm{L}$ Basta. We also transformed B. napus (Westar cultivar) hypocotyls with Agrobacterium bearing the pCAMBIA230135S:BnaFAX1-1 construct based on a protocol described previously [32]. We selected transgenic calli for growth on MS medium supplemented with Basta, and identified transgenic plants by PCR using BnaFAX1-1-specific primers (F35S3ND+BnaFAX1-1 RP (SacI), and BnaFAX1-1 FP (XbaI)+NOS5ND) (Additional file 1: Table S4). We then transferred positive 35S:BnaFAX1-1 transgenic plants to soil for seed setting and phenotyping.

\section{Subcellular localization of BnaFAX1-1-GFP fusion protein} We PCR-amplified the Arabidopsis FAX1 CDS from Arabidopsis Col-0 accession cDNA using the primer pair AtFAX1 FP (SpeI) + AtFAX1 RP (BamHI) (Additional file 1: Table S4). Similarly, we amplified the BnaFAX1-1 CDS by PCR from $B$. napus cDNA using the primer pair BnaFAX1-1 FP(SpeI) + BnaFAX1-1 RP (BamHI) (Additional file 1: Table S4). We subjected both PCR products to restriction digest with SpeI and BamHI before ligation into the pAN580 vector to generate pAN58035S:AtFAX1-GFP and pAN580-35S:BnaFAX1-1-GFP (Fig. 2A) [8], which were used to transiently transfect Arabidopsis protoplasts. We performed the transformation and analysis of Arabidopsis mesophyll protoplasts as described previously [10]. GFP fluorescence was detected at 672 to $750 \mathrm{~nm}$ and chlorophyll autofluorescence was monitored at 503 to $542 \mathrm{~nm}$ by laser scanning confocal microscopy (Carl Zeiss, LSM800). We used 35S:AtFAX1GFP as a positive control for the chloroplast inner envelope.

\section{Plant growth conditions and trait measurements}

For Arabidopsis, we surface-sterilized seeds from Col-0 and homozygous lines overexpressing BnFAX1-1 (BnaFAX1-1-OE) and sowed them on half-strength MS medium agar plates supplemented with $1 \%$ sucrose. We incubated plates in the dark at $4{ }^{\circ} \mathrm{C}$ for 2 days before releasing them in a plant incubator set to a light/dark photoperiod of $16 \mathrm{~h}$ light $/ 8 \mathrm{~h}$ dark. After 7 days, we transferred seedlings to soil and allowed them to grow in the incubator under the same conditions. After 7 weeks, we measured a number of phenotypes on all plants: stem fresh weight $(\mathrm{mg} / \mathrm{cm} ; 1 \mathrm{~cm}$ from the bottom of 2 nd internode of the primary inflorescence stem), stem diameter ( $\mathrm{mm}$; from bottom part of 2nd internode of the primary inflorescence stem), silique length, plant height, rosette fresh/dry weight, and fresh/dry weight of biological yield above ground per plant. We also collected the number of siliques per plant, the number of seeds per silique, the weight of 1000 seeds and the seed yield per plant after seeds maturation.

For $B$. napus growth in hydroponics, we germinated seeds for B. napus WT (Westar cultivar) and BnaFAX11-OE lines in glassware covered with three layers of wet filter paper. We transferred 8-day-old seedlings to quarter-strength Hoagland nutrient solution in a plant incubator under a temperature cycle of $26{ }^{\circ} \mathrm{C}$ (day)/22 ${ }^{\circ} \mathrm{C}$ (night) and a photoperiod of $16 \mathrm{~h} \mathrm{light/8} \mathrm{h}$ dark. We replaced the nutrient solution with half-strength Hoagland nutrient solution after 5 days, which we replaced 5 days later for full-strength Hoagland nutrient solution. We then refreshed the nutrient solution with full-strength Hoagland nutrient solution every 5 days until harvesting. After 32 days, we measured total root length, root area, root volume, root fresh/dry weight, leaf length, leaf width, leaf area, and leaf fresh/dry weight.

For field experimental work, we sowed B. napus WT (Westar cultivar) and BnaFAX1-1-OE lines in soil in Beibei, Chongqing $\left(29^{\circ} 45^{\prime} \mathrm{N}, 106^{\circ} 22^{\prime} \mathrm{E}, 238.57 \mathrm{~m}\right)$. Plants grown in field were arranged in a randomized field plot design with three replicates per genotype. Each plot contained four rows: 10 plants per row, $30 \mathrm{~cm}$ between rows, and $20 \mathrm{~cm}$ between plants within each row. After 175 days, we measured plant height, the number of leaves on the main stem for each plant, leaf length, leaf width, leaf area, the diameter at the same position on the main stem, chlorophyll content and photosynthetic rate for all plants. After seeds maturation, we scored plant height, effective branch number per plant (i.e., branches bearing seeds), length of the main inflorescence, silique number per main inflorescence, silique number per plant, silique length, seeds per silique, weight per 1000 seeds, overall seed yield per plant, and dry weight of biological yield above ground. We harvested 8-15 plants from each plot, averaged the measurements in each plot and used the resulting data for ANOVA.

\section{Plant phytohormone extraction}

We harvested 50-75 mg of leaf tissue from 35-day-old B. napus WT (Westar cultivar) and BnaFAX1-1-OE lines grown in incubator and extracted phytohormones using previously published methods [36]. Briefly, we mixed samples in 500-750 $\mu \mathrm{L} 2$-propanol/water/concentrated hydrochloric acid (2: 1: $0.002 \mathrm{v} / \mathrm{v} / \mathrm{v}$ ) for extraction and it was shaken at $100 \mathrm{rpm}$ for $30 \mathrm{~min}$ at $4{ }^{\circ} \mathrm{C}$. We then added $1 \mathrm{~mL}$ dichloromethane, and it also was shaken at $100 \mathrm{rpm}$ for $30 \mathrm{~min}$ at $4{ }^{\circ} \mathrm{C}$, then centrifuged the mixture and collected the supernatant. We repeated this extraction procedure three times, combined all supernatants and dried 
them under nitrogen flow. Finally, we added $200 \mu \mathrm{L}$ of methanol/0.1\% formic acid aqueous solution $(1: 1 \mathrm{v} / \mathrm{v})$ to resuspend the pellet, filtered it through an organic filter and placed each sample into the injection tube. We analyzed phytohormones by liquid chromatography followed by tandem mass spectrometry (LC-MS/SM) (QTRAP $6500+$ ) using the MRM approach described by Pan et al. [36] Briefly, LC uses a binary solvent system. The mobile phase is methanol and $0.05 \%$ formic acid. We selected an Eclipse plus C18 $(5 \mu \mathrm{m}, 2.1 * 150 \mathrm{~mm})$ chromatographic column. The flow rate was controlled at $300 \mu \mathrm{L} / \mathrm{min}$ and the column temperature was $30{ }^{\circ} \mathrm{C}, 10 \mu \mathrm{L}$ per injection. We used a gradient elution, with the initial gradient of methanol of $10 \%$, held for $2 \mathrm{~min}$, and gradually increased to $10 \mathrm{~min}$ and maintained at $90 \%$ for $5 \mathrm{~min}$. At $15.1 \mathrm{~min}$, we reduced methanol to the initial gradient and held for 7 min. We added external phytohormone standards for gibberellic acid, abscisic acid, indole-3-acetic acid, salicylic acid and jasmonic acid to calculate the level of each phytohormone in the samples.

\section{Lipid analyses}

We extracted lipids from 32-day-old leaves and analyzed lipid content by LC-MS/MS using the method reported previously [31]. We determined the levels and molecular species for phosphatidylcholine (PC), phosphatidylethanolamine (PE), phosphatidylglycerol (PG), phosphatidylinositol (PI), phosphatidylserine (PS), phosphatidic acid (PA), diacylglycerol (DAG) and triacylglycerol (TAG) as previously described [46]. For each experiment, we sampled six plants in six technical replicates; we used 50-100 mg fresh leaf tissue to extract lipids.

We quantified fatty acid composition using a gas chromatograph coupled with a flame ionization detector (GCFID). We immersed 3-5 mg dry seed samples in $1.5 \mathrm{~mL}$ methanol containing $1.5 \% \mathrm{H}_{2} \mathrm{SO}_{4}$ and $0.01 \%$ BHT. We then added $25 \mu \mathrm{L}$ heptadecanoic acid triglyceride (C17: 0 TAG) with $5.4 \mu \mathrm{mol} / \mathrm{L}$ in tubes as internal standard. We incubated the tubes in a water bath at $90^{\circ} \mathrm{C}$ for $1 \mathrm{~h}$ before allowing them to cool to room temperature. We then added $1 \mathrm{~mL} \mathrm{H}_{2} \mathrm{O}$ and $1 \mathrm{~mL}$ chromatographic hexane, mixed well, and centrifuged the samples at $1000 \mathrm{rpm}$ for $10 \mathrm{~min}$. Subsequently, we transferred $0.8 \mathrm{~mL}$ of the upper phase to a new glass tube and dried it under nitrogen flow. Finally, we added $0.4 \mathrm{~mL}$ hexane to dissolve fatty acid methyl esters, and injected $1 \mu \mathrm{L}$ of the ester solution into the $\mathrm{GC}$ with the detector temperature set to $280{ }^{\circ} \mathrm{C}$, oven temperature to $170{ }^{\circ} \mathrm{C}$ for $2 \mathrm{~min}$, and then increased by $3{ }^{\circ} \mathrm{C} / \mathrm{min}$ up to $210{ }^{\circ} \mathrm{C}$.

We determined total lipid content in seeds using a previously described method [33]. Briefly, we immersed $50 \mathrm{mg}$ dry seeds in $1 \mathrm{~mL}$ methanol (chromatography grade) and $2 \mathrm{~mL} 2 \%(\mathrm{~m} / \mathrm{v}) \mathrm{NaOH}$ in glass tubes, after which we mashed the seeds with glass rods and vortexed for $10 \mathrm{~min}$ on a vortex shaker. Next, we placed the glass tubes containing the seed mixture in a $60{ }^{\circ} \mathrm{C}$ water bath for $1 \mathrm{~h}$ before allowing the tubes to cool to room temperature. We then added $2 \mathrm{~mL}$ chloroform (chromatography grade) and vortexed again for $10 \mathrm{~min}$. After centrifugation of the mixture at $5000 \mathrm{~g}$ for $5 \mathrm{~min}$ at room temperature, we transferred the lower phase (chloroform) to a dry weighed glass tube, added $1 \mathrm{~mL}$ hexane (chromatography grade) to the remaining upper layer and vortexed again for $10 \mathrm{~min}$. After centrifugation, we collected the upper phase (hexane) and added it to the transferred chloroform solution. Finally, the mixture was dried under nitrogen flow and the content of total lipid was calculated. Five biological replicates were performed for each line.

\section{RNA isolation and quantitative RT-PCR analysis}

We extracted total RNA from various tissues of $B$. napus cultivar Zhongshuang11 (ZS11) using the EZ-10 DNAaway RNA Mini-prep Kit (Sangon Biotech (Shanghai), Co., Ltd). We then synthesized first-strand cDNAs from $1 \mu \mathrm{g}$ total RNA using the PrimeScript ${ }^{\mathrm{TM}} \mathrm{RT}$ reagent Kit with gDNA Eraser (Perfect Real Time) (TaKaRa Biotechnology, Dalian, China) according to the manufacturer's instructions. We performed real-time quantitative PCR analysis using SYBR Premix Ex Taq II (Perfect Real Time) (TaKaRa, Dalian, China) in a CFX96 real-time PCR system (Bio-Rad, USA) according to previous methods [28]. Gene-specific primers were designed using Vector NTI software, with the BnACTIN7 gene as internal reference gene (Additional file 1: Table S4) [7]. We performed RTqPCR on three independent biological replicates, each consisting of three technical replicates. We determined BnaFAX1-1 transcript levels in WT plants and transgenic lines by RT-qPCR as described above. In Arabidopsis, we used ACTIN2 as an internal control (Additional file 1: Table S4) [45].

\section{Statistical analysis}

All data were analyzed for statistical significance using SPSS 19.0 and GraphPad Prism 7. Analysis of variance was performed on data sets, and the values are presented as means $\pm \mathrm{SD}$. We used Duncan's test or Student's $t$ test to analyze the statistical significance between WT and transgenic lines $\left({ }^{*} P<0.05 ; * * P<0.01\right)$.

\section{Supplementary Information}

The online version contains supplementary material available at https://doi. org/10.1186/s13068-021-02035-4.

Additional file 1: Figure S1. Identification of new FAX genes in B. napus. We selected genes encoding chloroplast membrane proteins based on significant single-nucleotide polymorphisms (SNPs) associated with 
biological yield in rapeseed (13/14CQ-BY: 2013/2014Chongqing-biological yield). BnaFAX1-1 (BnaA07g17240D) was closely linked to the significant SNP Bn-A07-p12412116 for biological yield, according to our previous work [30]. Figure S2. Location, structure and conserved motif analysis of FAXs in B. napus. (A) The chromosomal distributions of the BnaFAX genes. (B) Gene structures of BnaFAXs and (C) conserved motifs analysis of BnaFAXs. Figure S3. Expression levels of six members of BnaFAX1 in six tissues of a pair of high- and low-seed oil content accessions grown in Chongqing (CQ24, CQ45) (A, B) and Yunnan (YN24, YN45) (C, D), and the expression levels of BnaFAX1-1 (E), BnaFAX1-2 (F) in seedling leaves (120 days in field) of four pair with high- (HBY) and low-biological yield accessions (LBY). CQ24, YN24 represent high-seed oil content accessions (HO); CQ45, YN45 represent low-seed oil content accessions (LO). St, Stem; Le, Leaf; 30ZP, silique pericarps on the main inflorescence of 30 days after flowering; $30 \mathrm{ZS}$, seeds on the main inflorescence of 30 days after flowering; 30CP, silique pericarps on the primary branch of 30 days after flowering; 30CS, seeds on the primary branch of 30 days after flowering. The expression levels of BnaFAX1-1 (E), BnaFAX1-2 (F) in seedling leaves (120 days in field) of four pair with high- (P281, P542, P125, P257-HBY) and low-biological yield accessions (P319, P276, P131, P81-LBY). The biological yield dry weight per plant for each accession (G). Figure S4. Phytohormone contents in leaves from 32-day-old WT and B. napus lines overexpressing BnaFAX1-1. ABA, abscisic acid; IAA, indole-3-acetic acid; SA, salicylic acid; JA, jasmonic acid. Table $\mathbf{S 1}$. Summary of significant associated SNPs and candidate genes for biological yield. Table S2. Chloroplast membrane proteins in candidate intervals associated with biological yield. Table S3. Identification of BnaFAX gene family members. Table S4. List of primer sequences used in this study.

\section{Acknowledgements}

We extend our thanks to the reviewers for their careful reading and helpful comments on this manuscript.

\section{Authors' contributions}

$N N L$, JNL and LG conceived and designed the experiments. ZCX, FT, LYZ, $S T L, S F W, Q H, B Y, C Z, L J W, C M Q, L G$ and KL performed the experiments and analyzed the data. ZCX and NNL wrote the paper. All authors reviewed the manuscript. All authors read and approved the final manuscript.

\section{Funding}

This study was funded by National Key R \& D Program of China (2016YFD0101900; 2018YFD0100504), Fundamental Research Funds for the Central Universities (XDJK2020B065, XDJK2018C095), the "111" Project (B12006), Modern Agro-industry Technology Research System (CARS-13), the National Natural Science Foundation of China (31870587; 31830067; 31400063; 31571701), Research Funds for Chongqing Talent (N. Li, 2019), Research Funds from State Key laboratory of Crop Stress Adaptation and Improvement (2020KF03), CAS Light of West China (XAB2018AW16).

\section{Availability of data and materials}

All data generated or analysed during this study are included in this published article and its supplementary information files.

\section{Declarations}

\section{Ethics approval and consent to participate}

Not applicable.

\section{Consent for publication}

Not applicable.

\section{Competing interests}

The authors declare that they have no competing interests.

\section{Author details}

${ }^{1}$ Chongqing Engineering Research Center for Rapeseed, College of Agronomy and Biotechnology, Southwest University, Chongqing 400715, China. ${ }^{2} \mathrm{Col}-$ lege of Resources and Environment, and Interdisciplinary Research Center for Agriculture Green Development in Yangtze River Basin, Southwest University, Chongqing 400715, China. ${ }^{3}$ Academy of Agricultural Sciences, Southwest University, Beibei, Chongqing 400715, China. ${ }^{4}$ College of Biology and Chemistry, Xingyi Normal University for Nationalities, Xingyi 562400, Guizhou, China. ${ }^{5}$ Hybrid Rapeseed Research Center of Shaanxi Province, Shaanxi, China. ${ }^{6}$ National Key Laboratory of Crop Genetic Improvement, Huazhong Agricultural University, Wuhan 430070, China. ${ }^{7}$ State Key Laboratory of Crop Stress Adaptation and Improvement, Key Laboratory of Plant Stress Biology, School of Life Sciences, Henan University, Kaifeng 475004, China.

Received: 21 June 2021 Accepted: 7 September 2021

Published online: 29 September 2021

\section{References}

1. Aili SR, Touchard A, Escoubas P, Padula MP, Orivel J, Dejean A, Nicholson $\mathrm{GM}$. Diversity of peptide toxins from stinging ant venoms. Toxicon. 2014;92:166-78.

2. Aulakh K, Durrett TP. The plastid lipase PLIP1 is critical for seed viability in diacylglycerol acyltransferase 1 mutant seed. Plant Physiol. 2019;180:1962-74.

3. Bailey TL, Williams N, Misleh C, Li WW. MEME: discovering and analyzing DNA and protein sequence motifs. Nucleic Acids Res. 2006;34:W369-373.

4. Cai G, Wang G, Kim SC, Li J, Zhou Y, Wang X. Increased expression of fatty acid and $A B C$ transporters enhances seed oil production in camelina. Biotechnol Biofuels. 2021;14:49.

5. Clough SJ, Bent AF. Floral dip: a simplified method for Agrobacteriummediated transformation of Arabidopsis thaliana. Plant J. 1998;16:735-43.

6. Deng XD, Gu B, Li YJ, Hu XW, Guo JC, Fei XW. The roles of acyl-CoA: diacylglycerol acyltransferase 2 genes in the biosynthesis of triacylglycerols by the green algae Chlamydomonas reinhardtii. Mol Plant. 2012;5:945-7.

7. Deng Z, Li X, Wang Z, Jiang Y, Wan L, Dong F, Chen F, Hong D, Yang G. Map-based cloning reveals the complex organization of the BnRf locus and leads to the identification of BnRf(b), a male sterility gene, in Brassica napus. Theor Appl Genet. 2016;129:53-64.

8. Du D, Liu M, Xing Y, Chen X, Zhang Y, Zhu M, Lu X, Zhang Q, Ling Y, Sang $X$, Li Y, Zhang C, He G. Semi-dominant mutation in the cysteine-rich receptor-like kinase gene, ALS1, conducts constitutive defence response in rice. Plant Biol. 2019;21:25-34.

9. Durrett TP, Benning C, Ohlrogge J. Plant triacylglycerols as feedstocks for the production of biofuels. Plant J. 2008;54:593-607.

10. Duy D, Wanner G, Meda AR, von Wiren N, Soll J, Philippar K. PIC1, an ancient permease in Arabidopsis chloroplasts, mediates iron transport. Plant Cell. 2007;19:986-1006.

11. Eriksson ME, Israelsson M, Olsson O, Moritz T. Increased gibberellin biosynthesis in transgenic trees promotes growth, biomass production and xylem fiber length. Nat Biotechnol. 2000;18:784-8.

12. Gasteiger E, Gattiker A, Hoogland C, Ivanyi I, Appel RD, Bairoch A. ExPASy: the proteomics server for in-depth protein knowledge and analysis. Nucleic Acids Res. 2003;31:3784-8.

13. Gracka A, Jelen HH, Majcher M, Siger A, Kaczmarek A. Flavoromics approach in monitoring changes in volatile compounds of virgin rapeseed oil caused by seed roasting. J Chromatogr A. 2016;1428:292-304.

14. Guo AY, Zhu QH, Chen X, Luo JC. GSDS: a gene structure display server. Yi Chuan. 2007;29:1023-6.

15. Hedden P. The current status of research on gibberllin biosynthesis. Plant Cell Physiol. 2020;61(11):1832-49.

16. Helliwell C, Sullivan JA, Mould RM, Gray JC, Peacock WJ, Dennis ES. A plastid envelope location of Arabidopsis ent-kaurene oxidase links the plastid and endoplasmic reticulum steps of the gibberellin biosynthesis pathway. Plant J. 2001;28(2):201-8.

17. Jones P, Binns D, Chang HY, Fraser M, Li W, McAnulla C, McWilliam H, Maslen J, Mitchell A, Nuka G, Pesseat S, Quinn AF, Sangrador-Vegas A, Scheremetjew M, Yong SY, Lopez R, Hunter S. InterProScan 5: genomescale protein function classification. Bioinformatics. 2014;30:1236-40.

18. Kim S, Yamaoka Y, Ono H, Kim H, Shim D, Maeshima M, Martinoia E, Cahoon EB, Nishida I, Lee Y. AtABCA9 transporter supplies fatty acids for lipid synthesis to the endoplasmic reticulum. Proc Natl Acad Sci USA. 2013;110:773-8. 
19. Kumar S, Stecher G, Tamura K. MEGA7: molecular evolutionary genetics analysis version 7.0 for bigger datasets. Mol Biol Evol. 2016;33:1870-4.

20. Li-Beisson Y, Shorrosh B, Beisson F, Andersson MX, Arondel V, Bates PD, Baud S, Bird D, Debono A, Durrett TP, Franke RB, Graham IA, Katayama K, Kelly AA, Larson T, Markham JE, Miquel M, Molina I, Nishida I, Rowland O, Samuels L, Schmid KM, Wada H, Welti R, Xu C, Zallot R, Ohlrogge J. Acyllipid metabolism. Arabidopsis Book. 2013;11:e0161.

21. Li N, Gugel IL, Giavalisco P, Zeisler V, Schreiber L, Soll J, Philippar K. FAX1, a novel membrane protein mediating plastid fatty acid export. PLoS Biol. 2015;13:e1002053.

22. Li N, Meng H, Li S, Zhang Z, Zhao X, Wang S, Liu A, Li Q, Song Q, Li X, Guo L, Li H, Zuo J, Luo K. Two plastid fatty acid exporters contribute to seed oil accumulation in Arabidopsis. Plant Physiol. 2020;182:1910-9.

23. Li NN, Xu CC, Li-Beisson YH, Philippar K. Fatty acid and lipid transport in plant cells. Trends Plant Sci. 2016;21:145-58.

24. Li NN, Zhang Y, Meng HJ, Li ST, Wang SF, Xiao ZC, Chang P, Zhang XH, Li Q, Guo L, Igarashi Y, Luo F. Characterization of fatty acid exporters involved in fatty acid transport for oil accumulation in the green alga Chlamydomonas reinhardtii. Biotechnol Biofuels. 2019;12:1-12.

25. Liu J, Hua W, Yang HL, Zhan GM, Li RJ, Deng LB, Wang XF, Liu GH, Wang HZ. The BnGRF2 gene (GRF2-like gene from Brassica napus) enhances seed oil production through regulating cell number and plant photosynthesis. J Exp Bot. 2012;63:3727-40.

26. Liu J, Hua W, Zhan G, Wei F, Wang X, Liu G, Wang H. Increasing seed mass and oil content in transgenic Arabidopsis by the overexpression of wri1like gene from Brassica napus. Plant Physiol Biochem. 2010;48:9-15.

27. Liu S, Fan C, Li J, Cai G, Yang Q, Wu J, Yi X, Zhang C, Zhou Y. A genomewide association study reveals novel elite allelic variations in seed oil content of Brassica napus. Theor Appl Genet. 2016;129:1203-15.

28. Lu K, Guo W, Lu J, Yu H, Qu C, Tang Z, Li J, Chai Y, Liang Y. Genome-wide survey and expression profile analysis of the mitogen-activated protein kinase (MAPK) gene family in Brassica rapa. PLoS ONE. 2015;10:e0132051.

29. Lu K, Peng L, Zhang C, Lu JH, Yang B, Xiao ZC, Liang Y, Xu XF, Qu CM, Zhang K, Liu LZ, Zhu QL, Fu ML, Yuan XY, Li JN. Genome-wide association and transcriptome analyses reveal candidate genes underlying yielddetermining traits in Brassica napus. Front Plant Sci. 2017;8:206.

30. Lu K, Xiao Z, Jian H, Peng L, Qu C, Fu M, He B, Tie L, Liang Y, Xu X, Li J. A combination of genome-wide association and transcriptome analysis reveals candidate genes controlling harvest index-related traits in Brassica napus. Sci Rep. 2016;6:36452.

31. Lu S, Sturtevant D, Aziz M, Jin C, Li Q, Chapman KD, Guo L. Spatial analysis of lipid metabolites and expressed genes reveals tissue-specific heterogeneity of lipid metabolism in high- and low-oil Brassica napus L. seeds. Plant J. 2018;94:915-32.

32. Lu SP, Bahn SC, Qu G, Qin HY, Hong Y, Xu QP, Zhou YM, Hong YY, Wang $X M$. Increased expression of phospholipase D1 in guard cells decreases water loss with improved seed production under drought in Brassica napus. Plant Biotechnol J. 2013;11:380-9.

33. Ma YB, Wang ZY, Zhu M, Yu CJ, Cao YP, Zhang DY, Zhou GK. Increased lipid productivity and TAG content in Nannochloropsis by heavy-ion irradiation mutagenesis. Bioresour Technol. 2013;136:360-7.
34. Miller JF, Zimmerman DC, Vick BA. Genetic-control of high oleic-acid content in sunflower oil. Crop Sci. 1987;27:923-6.

35. Nicolosi RJ, Woolfrey B, Wilson TA, Scollin P, Handelman G, Fisher R. Decreased aortic early atherosclerosis and associated risk factors in hypercholesterolemic hamsters fed a high- or mid-oleic acid oil compared to a high-linoleic acid oil. J Nutr Biochem. 2004;15:540-7.

36. Pan X, Welti R, Wang X. Quantitative analysis of major plant hormones in crude plant extracts by high-performance liquid chromatography-mass spectrometry. Nat Protoc. 2010;5:986-92.

37. Piazza GJ, Foglia TA. Rapeseed oil for oleochemical usage. Eur J Lipid Sci Tech. 2001;103:450-4.

38. Rudkowska I, Roynette CE, Nakhasi DK, Jones PJ. Phytosterols mixed with medium-chain triglycerides and high-oleic canola oil decrease plasma lipids in overweight men. Metabolism. 2006;55:391-5.

39. Talcott ST, Duncan CE, Del Pozo-Insfran D, Gorbet DW. Polyphenolic and antioxidant changes during storage of normal, mid, and high oleic acid peanuts. Food Chem. 2005:89:77-84

40. Tan H, Yang X, Zhang F, Zheng X, Qu C, Mu J, Fu F, Li J, Guan R, Zhang H, Wang G, Zuo J. Enhanced seed oil production in canola by conditional expression of Brassica napus LEAFY COTYLEDON1 and LEC1-LIKE in developing seeds. Plant Physiol. 2011;156:1577-88.

41. Tian Y, LV X, Xie G, Zhang J, Xu Y, Chen F. Seed-specific overexpression of AtFAX1 increases seed oil content in Arabidopsis. Biochem Biophys Res Commun. 2018;500:370-5.

42. Vigeolas H, Waldeck P, Zank T, Geigenberger P. Increasing seed oil content in oil-seed rape (Brassica napus L.) by over-expression of a yeast glycerol3-phosphate dehydrogenase under the control of a seed-specific promoter. Plant Biotechnol J. 2007;5:431-41.

43. Voorrips RE. MapChart: software for the graphical presentation of linkage maps and QTLs. J Hered. 2002;93:77-8.

44. Wang K, Froehlich JE, Zienkiewicz A, Hersh HL, Benning C. A plastid phosphatidylglycerol lipase contributes to the export of acyl groups from plastids for seed oil biosynthesis. Plant Cell. 2017;29:1678-96.

45. Wei Q, Hu P, Kuai BK. Ectopic expression of an Ammopiptanthus mongolicus $\mathrm{H}+$-pyrophosphatase gene enhances drought and salt tolerance in Arabidopsis. Plant Cell Tissue Organ Cult. 2012;110:359-69.

46. Welti R, Li WQ, Li MY, Sang YM, Biesiada H, Zhou HE, Rajashekar CB, Williams TD, Wang XM. Profiling membrane lipids in plant stress responsesrole of phospholipase D alpha in freezing-induced lipid changes in Arabidopsis. J Biol Chem. 2002;277:31994-2002.

47. Zhang H, Li M, He D, Wang K, Yang P. Mutations on ent-kaurene oxidase 1 encoding gene attenuate its enzyme activity of catalyzing the reaction from ent-kaurene to ent-kaurenoic acid and lead to delayed germination in rice. PLoS Genet. 2020;16(1):e1008562.

\section{Publisher's Note}

Springer Nature remains neutral with regard to jurisdictional claims in published maps and institutional affiliations.

Ready to submit your research? Choose BMC and benefit from

- fast, convenient online submission

- thorough peer review by experienced researchers in your field

- rapid publication on acceptance

- support for research data, including large and complex data types

- gold Open Access which fosters wider collaboration and increased citations

- maximum visibility for your research: over 100M website views per year

At BMC, research is always in progress.

Learn more biomedcentral.com/submissions 